University of Arkansas, Fayetteville

ScholarWorks@UARK

Graduate Theses and Dissertations

$12-2012$

\title{
Investigating the Sensitivity of Goodness-of-fit Indices to Detect Measurement Invariance in the Bifactor Model
}

Jam Khojasteh

University of Arkansas, Fayetteville

Follow this and additional works at: https://scholarworks.uark.edu/etd

Part of the Applied Statistics Commons

\section{Citation}

Khojasteh, J. (2012). Investigating the Sensitivity of Goodness-of-fit Indices to Detect Measurement Invariance in the Bifactor Model. Graduate Theses and Dissertations Retrieved from https://scholarworks.uark.edu/etd/610

This Dissertation is brought to you for free and open access by ScholarWorks@UARK. It has been accepted for inclusion in Graduate Theses and Dissertations by an authorized administrator of ScholarWorks@UARK. For more information, please contact scholar@uark.edu. 
INVESTIGATING THE SENSITIVITY OF GOODNESS-OF-FIT INDICES TO DETECT MEASUREMENT INVARIANCE IN THE BIFACTOR MODEL 
INVESTIGATING THE SENSITIVITY OF GOODNESS-OF-FIT INDICES TO DETECT MEASUREMENT INVARIANCE IN THE BIFACTOR MODEL

A dissertation submitted in partial fulfillment

of the requirements for the degree of

Doctor of Philosophy in Educational Statistics and Research Methods

By

\author{
Jam J. Khojasteh \\ Missouri State University \\ Bachelor of Science in Psychology, 2005 \\ Missouri State University \\ Master of Science in Experimental Psychology, 2008
}

December 2012

University of Arkansas 


\begin{abstract}
A Monte Carlo simulation study was conducted to evaluate the sensitivities of five commonly used goodness-of-fit indices to detect metric invariance properties of the bifactor model. The fit indices that performed the best in terms of power were $\Delta \mathrm{Gamma}$ and $\Delta \mathrm{Mc}$. In addition, $\Delta$ Gamma, $\Delta \mathrm{Mc}, \Delta \mathrm{CFI}$, and $\triangle \mathrm{RMSEA}$ all held Type I error to a minimum. However, only $\Delta$ Gamma and $\Delta \mathrm{CFI}$ are recommended to use in the bifactor model because the other $\Delta \mathrm{GOF}$ indices have cutoff values that are too large. For $\Delta$ Gamma and $\Delta$ CFI values of -.026 to -.045 and -.004 to -.009, respectively indicate a lack of metric invariance. In the variance component analysis, the magnitude of the factor loading differences contributed the most variation to each $\Delta \mathrm{GOF}$ except $\Delta \mathrm{SRMR}$. For $\Delta \mathrm{SRMR}$ the largest contribution of variance was model complexity (i.e., simple or complex). Finally, the Arkansas Benchmark Examination data was analyzed to compare the recommended cutoff criteria for $\Delta$ Gamma and $\Delta \mathrm{CFI}$ of the current study to the chisquare difference (likelihood ratio) test between configural and metric level invariance. The likelihood ratio test was consistent with $\Delta$ Gamma and $\Delta C F I$ for rejecting the test of metric invariance in the Arkansas Benchmark data.
\end{abstract}


This dissertation is approved for recommendation to the Graduate Council.

Dissertation Co-Chairs:

Dr. Wen-Juo Lo

Dr. Sean Mulvenon

Dissertation Committee:

Dr. Ronna Turner

Dr. George Marcoulides (ex officio) 


\section{DISSERTATION DUPLICATION RELEASE}

I hereby authorize the University of Arkansas Libraries to duplicate this dissertation when needed for research and/or scholarship

Agreed

Jam J. Khojasteh

Refused

Jam J. Khojasteh 


\section{ACKNOWLEDGMENTS}

I would like to thank God, my family, friends, and committee members. I am blessed to have such a supporting family who has, in addition, taught me skills and values that I will use and appreciate my whole life. Also, I want to thank my committee members who have provided me with valuable information and guidance during this process. Finally, I would like to thank each professor in our program. Each of you has influenced me in some aspect and made my educational experience enjoyable at the University of Arkansas. 


\section{DEDICATION}

To my family. 


\section{TABLE OF CONTENTS}

\section{LIST OF TABLES}

\section{LIST OF FIGURES}

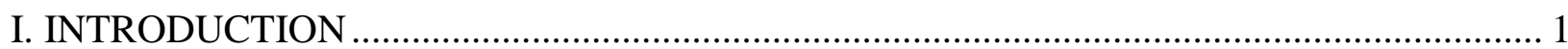

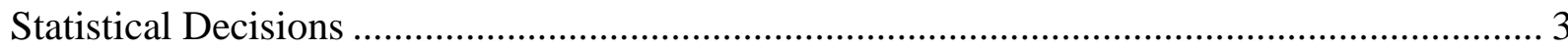

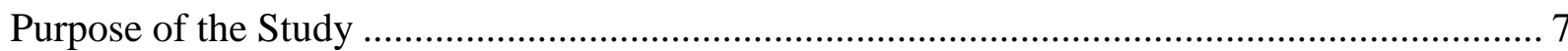

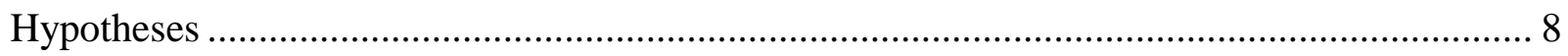

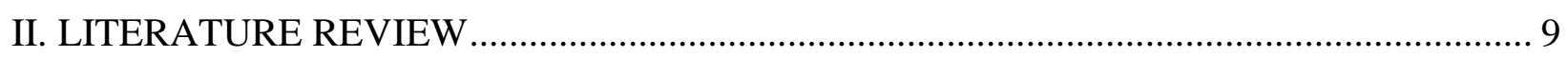

Measurement Invariance ................................................................................................. 9

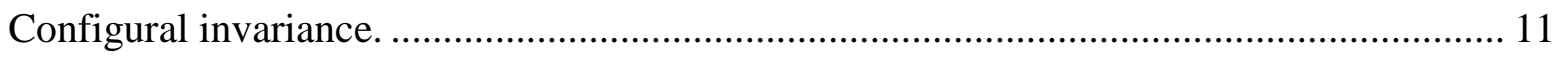

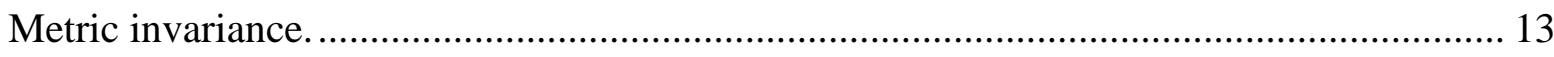

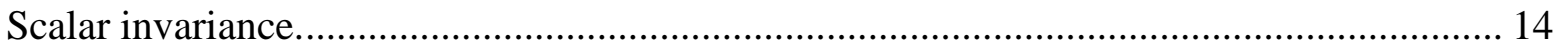

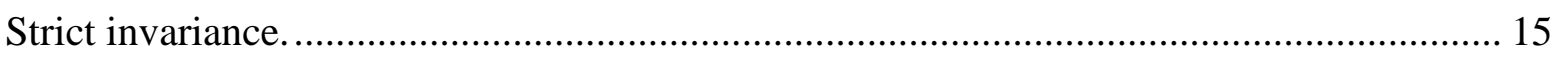

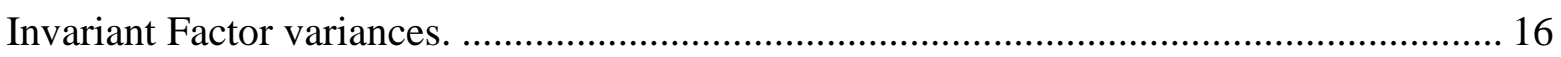

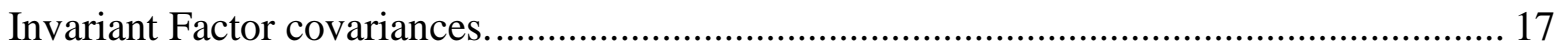

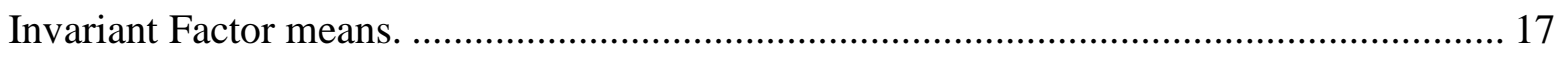

Goodness of Fit Indices Evaluated for the Current Study...................................................... 18

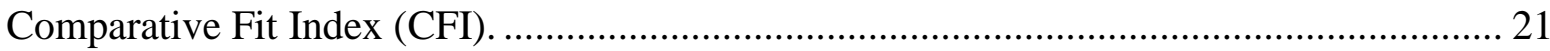

Root mean square error of approximation (RMSEA) ………......................................... 22

Standardized root mean square residual (SRMR). ............................................................ 23

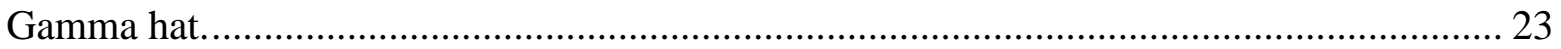

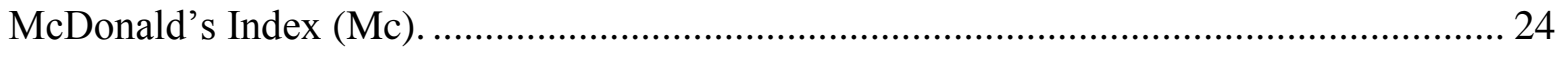

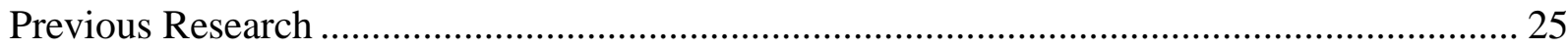

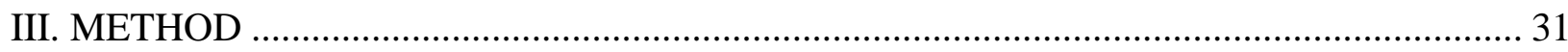

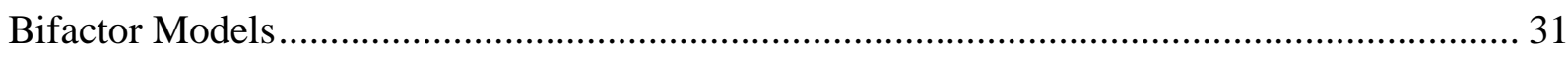

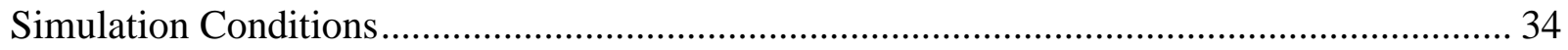

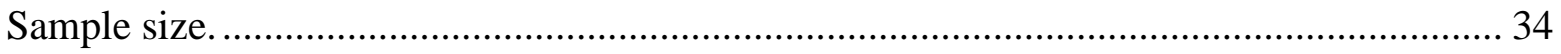

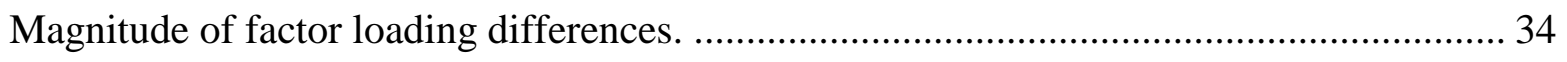

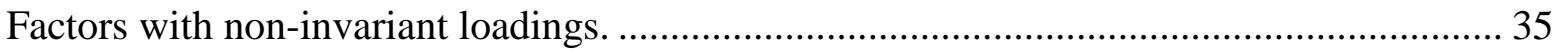

Percentage of specific factors with non-invariant items...................................................... 35 


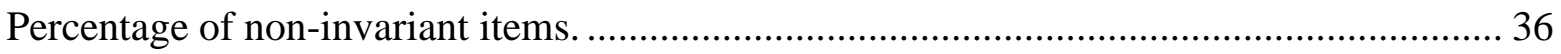

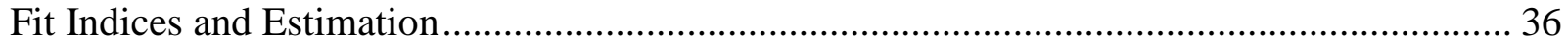

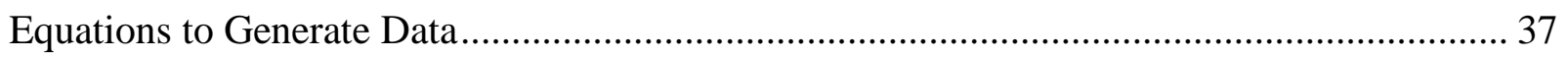

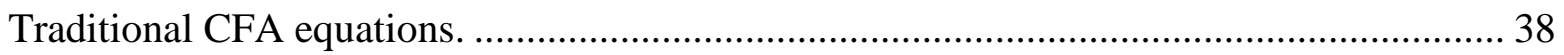

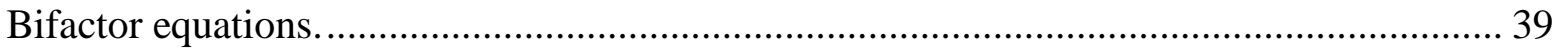

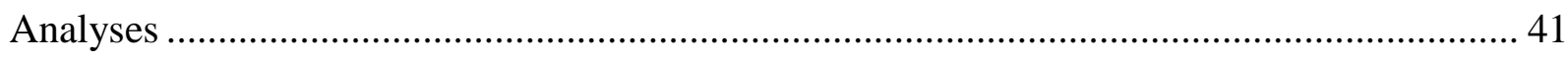

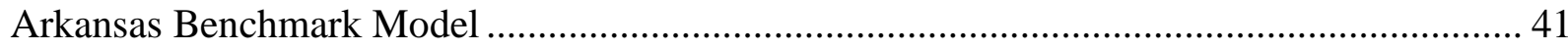

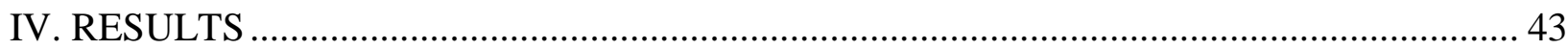

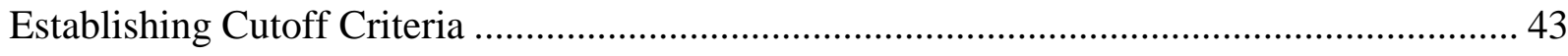

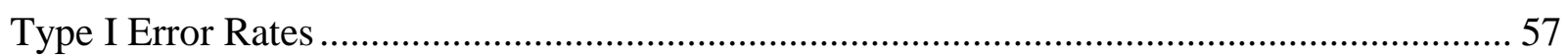

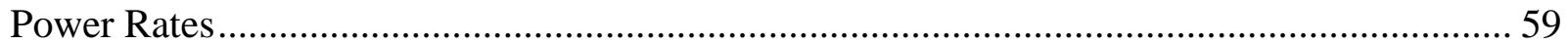

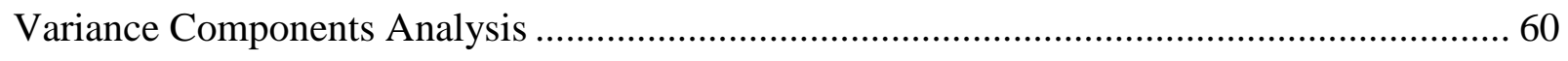

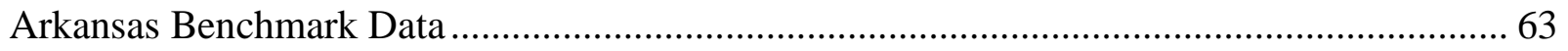

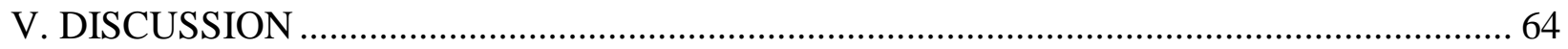

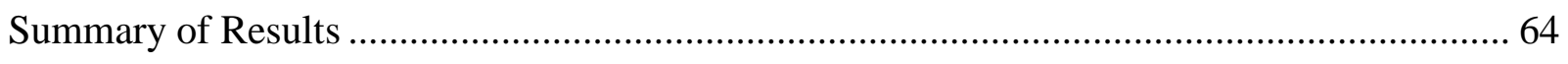

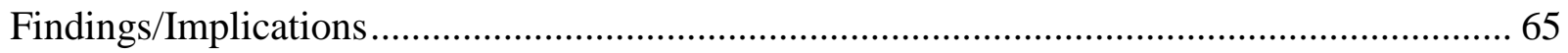

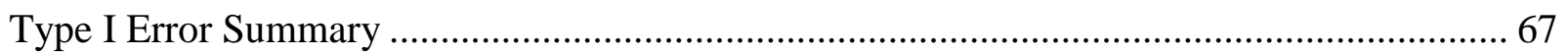

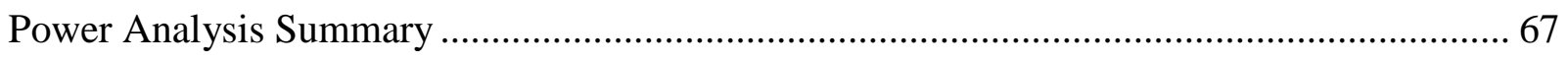

Recommendations for Future Research ...................................................................... 71

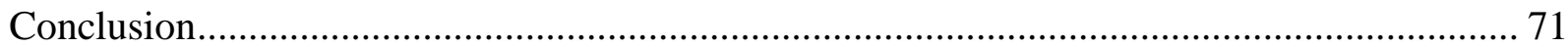

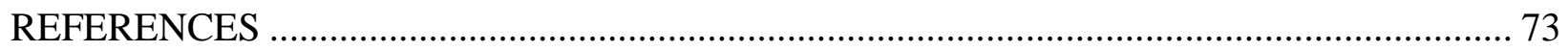




\section{LIST OF TABLES}

Table 1 Summary of Conditions for Simulation Study. 36

Table 2 Means, Standard Deviations, and $5^{\text {th }}$ Percentiles for $\Delta$ Gamma (Complex Model) by Condition... 45

Table 3 Means, Standard Deviations, and $5^{\text {th }}$ Percentiles for $\Delta$ Gamma (Simple Model) by Condition... 46

Table 4 Means, Standard Deviations, and $5^{\text {th }}$ Percentiles for $\Delta M c$ Donald's (Complex Model) by

Condition.. 47

Table 5 Means, Standard Deviations, and $5^{\text {th }}$ Percentiles for $\Delta$ McDonald's (Simple Model) by Condition. 48

Table 6 Means, Standard Deviations, and $5^{\text {th }}$ Percentiles for $\triangle C F I$ (Complex Model) by Condition.

Table 7 Means, Standard Deviations, and $5^{\text {th }}$ Percentiles for $\triangle C F I$ (Simple Model) by Condition

Table 8 Means, Standard Deviations, and 95 ${ }^{\text {th }}$ Percentiles for $\triangle S R M R$ (Complex Model) by Condition... 51

Table 9 Means, Standard Deviations, and 95 ${ }^{\text {th }}$ Percentiles for $\triangle S R M R$ (Simple Model) by Condition 52

Table 10 Means, Standard Deviations, and 95 ${ }^{\text {th }}$ Percentiles for $\triangle R M S E A$ (Complex Model) by Condition.

Table 11 Means, Standard Deviations, and 95 ${ }^{\text {th }}$ Percentiles for $\triangle R M S E A$ (Simple Model) by Condition. 54

Table 12 Simulation Cutoff Values by Model Complexity and Sample Size. 56

Table 13 Invariant Models' Means, Standard Deviations, and Percentiles to Yield Simulated

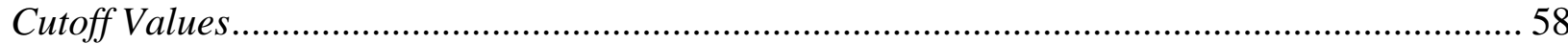

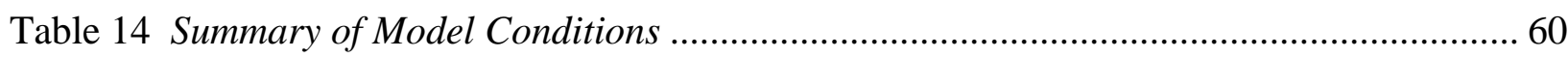

Table 15 Summary of Variance Component Analysis for Each $\triangle$ GOF Index .......................... 62

Table 16 Summary of Fit Indices for Arkansas Benchmark Data $(n=36,391)$.......................... 63

Table 17 Comparisons of Recommended Cutoff Criteria for $\triangle G O F$ Indices in the Bifactor Model Study Compared to Prior First-Order Studies. 66 


\section{LIST OF FIGURES}

Figure 1. Example of the bifactor model................................................................................. 6

Figure 2. Simple model used for simulation, one general and two specific factors................... 32

Figure 3. Complex model used for simulation, one general and four specific factors ................ 33 


\section{INTRODUCTION}

The concept of measurement invariance is the process of measuring individuals who come from different populations (i.e., different demographic information) to assess if they have the same likelihood of scoring equivalent to other populations on a particular construct or item, given the same latent ability (Meredith \& Millsap, 1992). In other words, the testing of measurement invariance properties evaluates whether different groups perceive and/or respond to the items the same. Measurement invariance is an important topic because if it fails to hold, group differences may be due to actual differences or just that different populations perceive a particular item differently (Millsap \& Kwok, 2004). Thus, interpretations become increasingly difficult or even inaccurate if proper measurement invariance properties have not been established. If measurement invariance holds then one can say the items on the questionnaire are invariant and both groups respond to the items the same. If measurement invariance does not hold then one cannot say that the two groups perceive the items the same, thus further analyses comparing the two groups should be cautioned or even disregarded. That is, if the two groups are not perceiving and answering the questions the same, how can a researcher conduct further analyses between the two groups? If further analyses are conducted, the interpretations can be biased and invalid, because the differences between the groups cannot be differentiated between "true" differences or due to the two groups perceiving the items differently (Millsap \& Kwok, 2004).

Measurement invariance (MI) is analogous to traditional differential item functioning (DIF) analyses. Therefore, evaluation of MI properties can be conducted in a traditional Item Response Theory (IRT) framework or a Structural Equation Modeling (SEM) framework which is a technique used to measure latent constructs as well as relationships between these constructs 
(Bollen, 1989). One perspective of SEM can be described as confirmatory factor analysis (CFA) and path analysis combined into one theoretical model where, the CFA component of the model is the measurement model and the path analysis part is considered the structural model (Bollen, 1989). In general, SEM can be used as an ideal procedure to evaluate how well a researcher's theory (i.e., theoretical model) fits a sample's variance-covariance matrix. Using estimation methods, such as maximum likelihood (ML), the difference between the sample variancecovariance matrix and model-implied variance-covariance matrix (i.e., variances and covariances reproduced from the hypothesized model) is minimized. Equation 1.1 is the hypothesis that the sample and model implied matrices are equal, where $\Sigma$ (sigma) is the population matrix (represented by a random sample of the population) of observed variables, $\theta$ (theta) contains the model parameters, and $\Sigma(\theta)$ is the model-implied variance-covariance matrix (Bollen, 1989).

$$
\Sigma=\Sigma(\theta)
$$

If a hypothesized theory fits the data well (i.e., the residuals between the sample and modelimplied variance-covariance matrix are minimal), the researcher can support the hypothesis that this model is one of the true plausible models in the population. However, one caution of SEM is that the researcher can only support the proposed theoretical model and never prove it. The data may fit the model well, but one cannot say that the hypothesized model is the true population model. Conversely, if the model is rejected (i.e., the hypothesized model does not fit the data), then that model can be ruled out as one of the plausible population models or perhaps the model was misspecified (Bollen, 1989; Kline, 2011). Thus, when conducting multigroup confirmatory factory analyses (MCFA), the issue of MI will be important to address to determine if the groups perceive a questionnaire or assessment similarly. 
When conducting tests of MI properties, one first needs to define a theoretical model to evaluate along with the groups that will be included in the analyses. Then successive CFA models (each imposing more restrictions) are compared to a null model with no constraints or a model with fewer constraints to determine if the different types of constraints impact the fit of the models. The first step of the MI analysis is configural invariance which tests if the pattern of fixed and free parameters is the same for the groups. If configural invariance does not hold then testing of MI properties must stop and further analyses should not be conducted. The next step evaluates if the factor loadings are invariant across groups and is denoted here as metric invariance. Subsequently, the test of scalar invariance is used to determine if the item intercepts (i.e., means) are invariant. Finally, the last step is strict invariance which tests for invariance of the items' error variances (Millsap \& Kwok, 2004). The theoretical models proposed in the SEM framework are evaluated using fit indices and are described next.

\section{Statistical Decisions}

When researchers began using covariance modeling (i.e., structural equation modeling and confirmatory factor analysis) the chi-square $\left(\chi^{2}\right)$ test was used to evaluate the null hypothesis that the population and model-implied covariances were equal, that is, perfect fit (Gerbing \& Anderson, 1993). However, the issue of sample size and statistical significance soon became evident (Gerbing \& Anderson, 1993). Therefore, with large sample sizes, minor deviations between the sample and model implied parameters may lead to model rejection (i.e., concluding that the model did not fit the data) (Gerbing \& Anderson, 1993; Millsap, 2007). Consequently, to counteract the issue of sample size and power, researchers began to formulate alternative fit indices (Bentler \& Bonett, 1980; Jöreskog \& Sörbom, 1981). The three major types of indices (i.e., absolute, incremental, and parsimonious) are briefly described below. 
The first type of fit indices described can be characterized as overall model fit, which assesses the residuals between the predicted (model-implied) and observed (population) covariance matrices. The Goodness-of-Fit Index (GFI) is one of the most well-known indices to evaluate this relationship (Jöreskog \& Sörbom, 1981). The second type of fit indices assesses the relationship between two nested models, usually the null model and a more restrictive model. When evaluating MI properties, the null model allows for the parameters to vary across populations whereas, the restrictive model constrains the parameters to be equal across populations. The null model serves as a baseline for the comparison of incremental fit for the model of interest (Gerbing \& Anderson, 1993). That is, the null model (i.e., free estimation of all parameters) is usually not of theoretical importance, whereas, the more restrictive model (i.e., the specified model) is theoretically more meaningful. Thus, the incremental fit is used to see if the theoretically meaningful model fits the data better than the null model, such as Bentler and Bonett's (1980) Normed fit index (NFI) and Tucker and Lewis' (1973) Tucker-Lewis index (TLI).

Anderson and Gerbing (1988) stated that SEM can be thought of as a comparative technique in which a hypothesized model is compared against rival, theoretically alternative models. However, one problem that can arise with these types of fit indices is that model fit can be improved simply by freeing more parameters until the degrees of freedom $(d f)$ is equal to zero and a "perfect fit" is found (Gerbing \& Anderson, 1993). Therefore, the next set of fit indices takes into account the degrees of freedom and adjusts accordingly. The third set of fit indices are based on model complexity where parsimonious models (i.e., adjusted for the $d f$ of the new model) are rewarded for having more degrees of freedom (Gerbing \& Anderson, 1993). These fit indices include Jöreskog and Sörbom's (1988) Adjusted Goodness of Fit Index (AGFI) and 
Steiger and Lind's (1980) and Steiger's (1989) Root Mean Square Error of Approximation (RMSEA). The purposes of fit indices in simulation studies can be used to evaluate many SEM and CFA issues, in particular measurement invariance (Chen, 2007; Cheung \& Rensvold, 2002; French \& Finch, 2006; Fan \& Sivo, 2009). Provided below is a discussion of how to evaluate competing models, beginning with the likelihood ratio (LR) test, followed by a brief review of the literature that was used to formulate the study.

The LR test can be used to compare nested models to investigate the measurement invariance properties across populations by computing a chi-square difference test between the two models (Bollen, 1989). However given the strict assumptions of the LR test (see Chapter 2 for details), there have been a few studies that have investigated alternative goodness of fit (GOF) indices under the measurement invariance framework (e.g., Chen, 2007; Cheung \& Rensvold, 2002; French \& Finch, 2006; Fan \& Sivo, 2009). Cheung and Rensvold (2002) reviewed 20 different delta goodness-of-fit ( $\Delta \mathrm{GOF})$ indices when conducting two group measurement invariance tests. Chen (2007) elaborated further on Cheung and Rensvold's (2002) study by conducting two Monte Carlo studies to evaluate MI and common $\Delta \mathrm{GOF}$ indices. French and Finch (2006) investigated MI properties using two fit indices (i.e., $\chi^{2} \&$ CFI). More recently, Fan and Sivo (2009) elaborated further on the issues of $\Delta \mathrm{GOF}$ indices by assessing mean structure invariance. However, all of the above studies were designed and evaluated for the firstorder model. To my knowledge, using the bifactor model and assessing $\Delta \mathrm{GOF}$ indices for measurement invariance across populations has not been evaluated, thus, leaving an uncertain area of research in the MI literature.

The bifactor model was first proposed by Holzinger and Swineford (1937) which consists of specific latent factors and a general latent factor for a group of items. One example of the 
bifactor model includes method effects, where researchers can evaluate the wording effects of specific items (i.e., positively and negatively worded items) (DiStefano \& Motl, 2006). Another example of the bifactor model can be illustrated using intelligence. Suppose an IQ test has three specific factors (with 3 items each) that are assumed to measure certain abilities (i.e., spatial, verbal, and quantitative), however, a "general" intelligence ability also exist. Therefore, the nine item test has four latent factors, three specific (also known as group factors) and one general factor. An illustrated example is presented below in Figure 1.

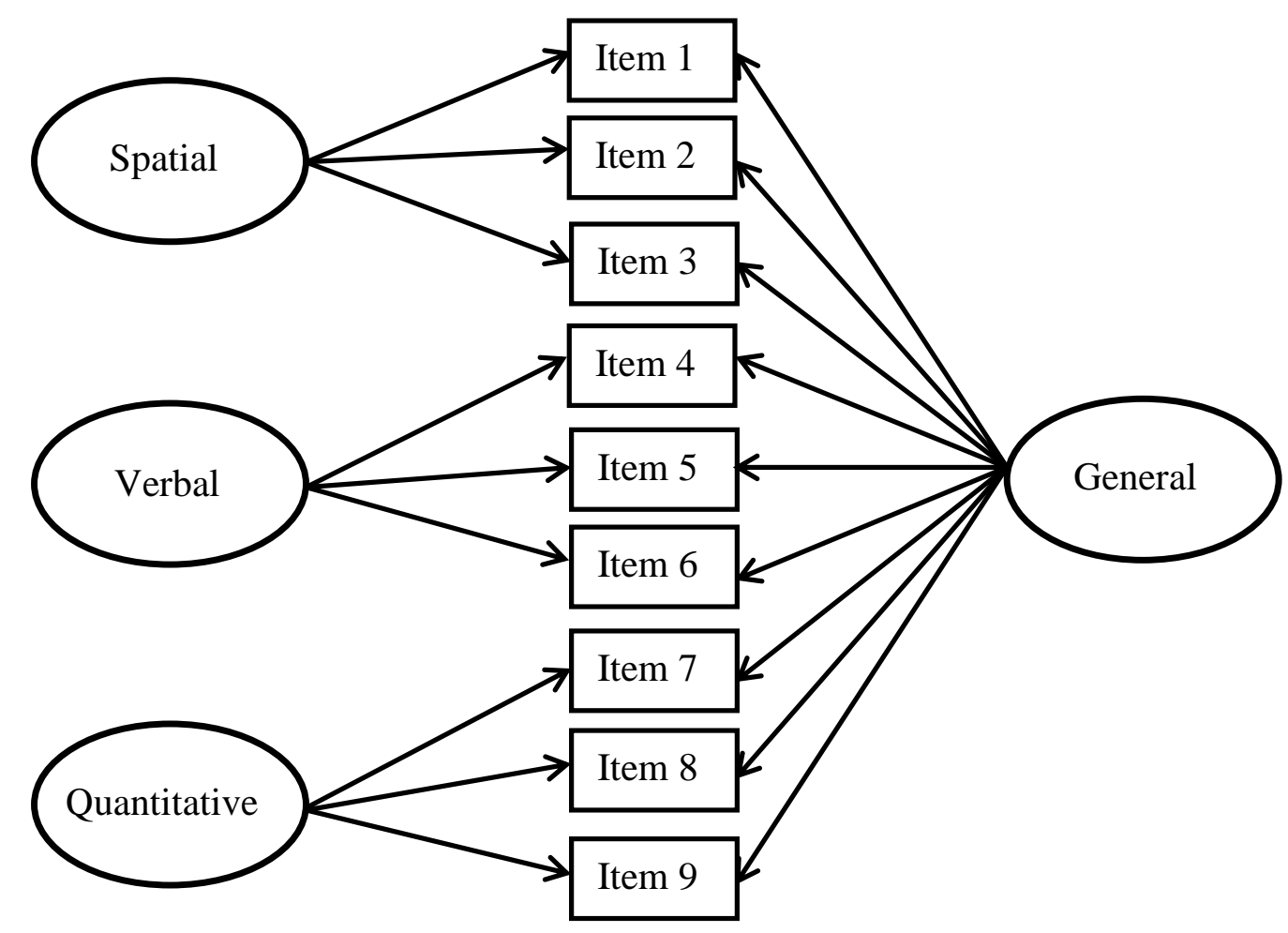

Figure 1. Example of the bifactor model.

One advantage of the bifactor model is that it can be used to study the domain specific factors independent of the general factor, thus measurement invariance properties of the specific 
and general factors can also be assessed (Chen, West, \& Sousa, 2006). That is, substantive researchers can evaluate the factor loadings on both the general and specific domain factors to evaluate the invariance of items for multiple groups. In applied studies, the bifactor model and other higher order models have not been used until recently (Chen, West, \& Sousa, 2006). Chen, West, and Sousa (2006) specified that some of the most recent use of the bifactor model has been in the area of intelligence research (e.g., Gustafsson \& Balke, 1993; Luo, Petrill, \& Thompson, 1994). Given that the bifactor model has mostly been used by applied researchers, in particular intelligence studies, the measurement invariance properties of the bifactor model should also be assessed. In addition, Chen, West, and Sousa (2006) advocate the use of the bifactor model, which in turn, may lead to more applied research using this model. Therefore, it is necessary to investigate the sensitivity of common GOF indices for testing MI properties using the bifactor model.

\section{Purpose of the Study}

A Monte Carlo simulation study will be conducted to evaluate the sensitivity of $\Delta \mathrm{GOF}$ indices across groups when investigating measurement invariance properties using the bifactor model. According to Cheung and Rensvold's (2002) simulation study, $\Delta \mathrm{CFI}, \Delta \mathrm{Gamma}$ hat, and $\Delta \mathrm{McDonald}$ 's fit indices are recommended because they are not redundant with other $\Delta \mathrm{GOF}$ indices (i.e., not highly correlated), not affected by sample size, and are robust for testing between-group invariance. Chen (2007) investigated five fit indices which were $\Delta$ CFI, $\triangle \mathrm{RMSEA}, \Delta \mathrm{SRMR}, \Delta \mathrm{Gamma}$ hat, and $\Delta \mathrm{McD}$ onald's index. She recommended $\Delta \mathrm{CFI}$, $\triangle \mathrm{RMSEA}$, and $\triangle \mathrm{SRMR}$ because $\Delta \mathrm{Gamma}$ hat was highly correlated with $\Delta \mathrm{CFI}$ (contrary to Cheung \& Rensvold, 2002) and $\triangle$ RMSEA and $\triangle$ SRMR performed just as well as $\Delta$ McDonald's. In addition, Chen (2007) prefers these $\Delta \mathrm{GOF}$ indices because they are some of the most 
commonly used indices in the literature. Recently, Meade, Johnson, and Braddy (2008)

conducted a simulation study comparing 20 fit indices, similar to Cheung and Rensvold's (2002)

study, and the results conferred that $\Delta \mathrm{CFI}$ and $\Delta \mathrm{McDonald}$ 's index provided adequate power and sensitivity to detect non-invariant items. Using these studies as a guide, all five indices (i.e., $\triangle \mathrm{CFI}, \triangle \mathrm{RMSEA}, \triangle \mathrm{SRMR}, \Delta \mathrm{Gamma}$ hat, and $\Delta \mathrm{McDonald}$ 's index) will be compared in the current study to investigate their effectiveness when using a bifactor model.

\section{Hypotheses}

In this study, the sensitivity of five $\triangle \mathrm{GOF}$ indices (i.e., $\triangle \mathrm{CFI}, \triangle \mathrm{RMSEA}, \triangle \mathrm{SRMR}$, $\Delta$ Gamma hat, and $\Delta \mathrm{McDonald}$ 's) will be compared for evaluating MI properties using the bifactor model. Specifically, indices which perform the best in terms of Type I error and power (i.e., more robust) for each $\Delta \mathrm{GOF}$ under varying conditions will be identified. The study will provide applied researchers with guidelines for $\triangle \mathrm{GOF}$ indices when investigating measurement invariance using the more complex bifactor model, as compared to first-order models, and hopefully stimulate more research in the area of measurement invariance. 


\section{LITERATURE REVIEW}

To familiarize the reader with the current study, a review of measurement invariance (MI) is provided that will detail the sequential process used in MI testing along with the different types of MI tests (i.e., configural, metric, scalar, and strict). The next section will summarize the selected delta goodness-of-fit ( $\Delta \mathrm{GOF}$ ) indices that will be investigated in the current study and also provide the equations, recommended cutoff values, and the type of index it is considered (i.e., absolute, incremental, or parsimonious). The following section will discuss previous research investigating the sensitivities of particular $\triangle \mathrm{GOF}$ indices for MI properties. Finally, a summary of the bifactor model will be provided.

\section{Measurement Invariance}

Measurement Invariance testing and its associated properties are important for researchers to consider when comparing groups on a particular test or assessment. In other words, when making group comparisons on a specific measure, a researcher must first determine or have previous support that the two groups perceive the items the same. If MI fails to fully hold then further investigation into group differences may be problematic. However, if some but not all of the parameters are invariant then partial invariance exists (Byrne, Shavelson, \& Muthén, 1989; Millsap \& Kwok, 2004). MI under the confirmatory factor analysis (CFA) framework, which is the most widely used tool to investigate MI, involves sequential testing of increasingly restrictive models to evaluate if certain parameters are invariant (e.g., item loadings, intercepts, and errors) (Byrne, Shavelson, \& Muthén, 1989; Jöreskog, 1971; Meredith, 1993; Millsap \& Everson, 1993; Reise, Widaman, \& Pugh, 1993; Vandenburg, 2002; Vandenburg \& Lance, 2000). A test or measure is considered invariant when individuals from a different population who are identical on the construct being measured have the same probability of achieving a 
given score on the test or assessment (Meredith \& Millsap, 1992). If a test is not invariant, then individuals who are from different populations, but have the same latent ability, could be expected to score differently (Millsap \& Kwok, 2004). For example, let X be a $p$ x 1 vector of measured variables, W represented as an $r \times 1$ vector of latent variables, and $\mathrm{V}$ an $s \times 1$ vector of population indicators that includes information to distinguish different groups. If the probability of $\mathrm{X}$ given $\mathrm{W}$ is the same regardless of group membership $\mathrm{V}$, then $\mathrm{X}$ fulfills measurement invariance in relation to $\mathrm{W}$ and $\mathrm{V}$ (Millsap, 2005).

$$
P(\mathrm{X} \mid \mathrm{W}, \mathrm{V})=P(\mathrm{X} \mid \mathrm{W})
$$

Millsap (2005) states that “...invariance of $\tau, \lambda$, and $\Theta$ (scalar, metric, and strict invariance, respectively) is a necessary, but not sufficient condition for measurement invariance, in addition, measurement invariance requires consideration of the distributional form for $P(\mathrm{X} \mid \mathrm{W}, \mathrm{V})$ and whether this distribution depends on V" (p. 155). In other words, the parameters must be invariant as well as the distributional form of $\mathrm{X}$ given the latent factor scores.

The simplest case of measurement invariance consists of two groups, the reference group, usually the majority or higher scoring group, and focal group, which is assumed to be negatively affected by any lack of invariance (Holland \& Thayer, 1988). For measurement invariance to hold, the participants who have the same ability from both groups should have the same observed score, $\mathrm{X}_{\mathrm{ik}}$ which represents a $p \times 1$ vector of scores on $p$ measured items for the $i$ th person in the $k$ th group. The measured items should fit a common factor model (i.e., both groups have the same CFA model) to yield the following equation:

$$
X_{i k}=\tau_{k}+\lambda_{k} \xi_{i k}+\delta_{i k}
$$


where $\tau_{\mathrm{k}}$ is a $p \times 1$ vector of item intercepts in the $k$ th group, $\lambda_{\mathrm{k}}$ is a $p \times 1$ vector of factor loadings (regression slopes related to $\mathrm{X}$ ), $\xi_{\mathrm{ik}}$ is the latent factor score for the $i$ th person in the $k$ th group, and $\delta_{i k}$ is a $p \times 1$ vector of unique factor variances for the $i$ th person in the $k$ th group. Assuming a standard factor analytic model where $E(\xi, \delta)=0$, the above equation implies that the covariance matrix sigma $\left(\Sigma_{\mathrm{k}}\right)$ and mean vector $\left(\mu_{\mathrm{k}}\right)$ for $\mathrm{X}_{\mathrm{ik}}$ are

$$
\begin{aligned}
& \Sigma_{k}=\Lambda_{k} \Phi \Lambda_{k}^{\prime}+\Theta_{k} \text { and } \\
& \mu_{k}=\tau_{k}+\lambda_{k} \kappa_{k}
\end{aligned}
$$

where $\Lambda$ is a $p \times n$ (where $n$ is the number of latent factors) matrix of factor loadings linking the latent and manifest variables, $\Phi$ is a $n$ x $n$ matrix of the common factor variances and covariances for $\xi, \Theta$ is a $p \times p$ diagonal matrix of unique factor variances (assuming no correlated error terms), and $\kappa$ is the common factor mean in the $k$ th group. The equations presented above demonstrate the mathematical relationships between the parameters and their latent and manifest counterparts within a CFA framework. The above equations will be used to explain MI in mathematical terms and the associated MI concepts. Provided below is a detailed summary of each sequential step in testing for measurement and structural invariance. The first four steps test aspects of measurement invariance, which is concerned with the relationship between measured variables and latent factors (i.e., the measurement model). The last three steps are concerned with structural invariance, which consist of evaluating the relationships between the latent factors (i.e., the structural model).

Configural invariance. First, both groups must be evaluated to assess if they have the same common factor structure. The omnibus test of equality of covariance matrices across groups must hold before further MI testing begins. That is, $\xi_{k}=\xi_{k^{\prime}}$ where $k$ and $k^{\prime}$ represent 
different groups. The overall null hypothesis of equal factor structure by groups is evaluated using the chi-square test statistic or other GOF indices. Failing to reject the overall null hypothesis of group equivalence indicates that the two groups share the same common factor structure and has two main implications. The first implication is that the responding groups both perceived the items from the same frame of reference and therefore can be compared. The second implication is that further MI testing can be performed as long as the sequential tests of MI (i.e., each MI test imposes more restrictions) are nested within the test of configural invariance (Vandenburg \& Lance, 2000). However, rejecting the null hypothesis of configural invariance indicates that the two groups do not share the same factor structure and further MI testing should be precluded. Conceptually, it makes no sense to continue with MI testing when the constructs are perceived differently across groups. That is, the groups are treating the underlying constructs differently and therefore group comparisons or further MI testing is problematic (Vandenburg \& Lance, 2000).

Meade, Johnson, and Braddy (2008) provided a succinct discussion of configural invariance that researchers should also consider. They indicated that poor model fit might be due to one of two things. First, the model may fit the data well for one group but not the other, which is evidence that configural invariance does not hold. Second, the model may fit the data poorly for both groups, which may indicate that the model is not properly specified. Therefore, Meade, Johnson, and Braddy (2008) proposed a slightly different approach to configural invariance testing which involved testing the CFA model in each group separately. If the model fits the data well for each group, then the researchers can evaluate the CFA model using the combined data (i.e., aggregated over groups) which serve as a baseline model for further MI testing. If the model does not fit the data well in the groups separately, then Meade, Johnson, and Braddy 
(2008) suggests using either exploratory factor analysis (EFA), Lagrange multipliers (LM), or Wald tests to aid in properly specifying the CFA model. However, changes made to the model based on modification indices (e.g., LM or Wald tests) should be theoretically justified and specified not only on a post hoc basis (Bollen, 1989).

Metric invariance. This test evaluates if the factor loadings $(\lambda)$ are invariant across the groups, by constraining the loadings of the same items to be equal across groups. The factor loadings are the regression slopes of $\mathrm{X}_{\mathrm{ik}}$ to their respective latent factors $(\xi)$ and represent an expected change in observed score given a one unit increase in the latent variable (Bollen, 1989). Therefore, the equality test of scaling units across groups is represented by the null hypothesis, $\Lambda_{\mathrm{k}}=\Lambda_{k^{\prime}}$. If this test holds then all the factor loadings are invariant across groups. For the current study this is denoted as metric invariance (Horn \& McArdle, 1992). However, other terms proposed for this condition are weak factorial invariance by Widaman and Reise (1997) or pattern invariance by Millsap (1997). If some but not all of the $p$ factor loadings are invariant then partial invariance may still hold (Byrne, Shavelson, \& Muthén, 1989; Millsap \& Kwok, 2004). However, there has been some disagreement in the literature about partial metric invariance (Vandenburg \& Lance, 2000). For example, Bollen (1989) indicated that rejecting the null hypothesis of metric invariance should preclude any further testing of MI (same as configural invariance testing), whereas, Byrne et al. (1989) have encouraged the search for partial metric invariance. In response to the disagreement, Vandenburg and Lance (2000) offer further insight into the use of continuing with partial invariance providing both an advantage and disadvantage. The advantage of testing for partial invariance is that statistical criteria (i.e., GOF indices) are not applied consistently throughout the literature (i.e., cutoff values) to reject or fail to reject full invariance. Therefore, if a model has adequate fit and cutoff criteria are applied 
inconsistently, partial invariance should be investigated to evaluate the possibility of some invariant items, not just completely invariant or non-invariant. However, a disadvantage is that partial invariance constraints are post hoc in nature, capitalize on chance, and may not be theoretically meaningful to impose. Therefore, Vandenburg and Lance (2000) recommend a conservative approach to partial invariance testing where metric invariance is only relaxed for a small subset of the indicators, backed by a strong theoretical rationale, and uses cross-validation evidence when possible (other means of verification) to support the rationale of relaxing constraints for certain items.

Scalar invariance. The test of $\tau_{\mathrm{k}}=\tau_{k^{\prime}}$ evaluates if the item intercepts $(\tau)$ are invariant across groups, in addition to the test of metric invariance.

$$
\lambda_{k}=\lambda \quad \text { and } \quad \tau_{k}=\tau \text { for all } k
$$

The item intercepts represent the value of an observed item when the value of the corresponding factor $(\xi)$ is zero, and is one of the least used tests of MI (Vandenburg \& Lance, 2000). The invariance tests of intercepts can represent two things. First, intercept differences may reflect an undesirable response bias, or they may represent group mean differences, which can be appropriate given the substantive research question (Vandenburg \& Lance, 2000). For example, given a particular measure it is expected that males have a higher score on the construct than females and a hypothesis that states males perform higher should be true, given this particular measure. Therefore, a test of scalar invariance is not appropriate because differences between the two groups would be expected and a test of invariance would be conceptually meaningless. Another term for scalar invariance is strong factorial invariance proposed by Meredith (1993) 
and like partial metric invariance, partial scalar invariance occurs when some, but not all intercepts are invariant (Millsap \& Kwok, 2004).

Strict invariance. The last sequential test in MI is strict invariance, $\Theta_{\delta \mathrm{k}}=\Theta_{\delta k^{\prime}}$ which tests the invariance of the unique variances (and possibly covariances) across groups and occurs when all loadings, intercepts, and unique item variances are invariant (Meredith, 1993; Vandenburg \& Lance, 2000) as defined below.

$$
\lambda_{k}=\lambda, \quad \tau_{k}=\tau, \quad \text { and } \quad \delta_{k}=\delta \text { for all } k
$$

Usually $\Theta_{\delta}$ is specified as a diagonal matrix of item variances where all covariances are equal to zero. However, correlated errors are sometimes estimated or should be estimated when conducting studies over time (e.g., repeated measures) (Ferron, Dailey, \& Yi, 2002; Grimm \& Widaman, 2010; Kwok, West, \& Green, 2007; Sivo, Fan, \& Witta, 2005). One may wonder why this issue is important to investigate, especially when one of CFA's assumptions is that errors are $N \sim\left(0, \sigma^{2}\right)$ (i.e., normally distributed with a mean of zero and a specified variance) and in addition, no error covariances exist (Bollen, 1989). However, many CFA models are specified with correlated error variances (i.e., error covariances) for either theoretical reasons or based on modification indices, which the latter are usually not implemented based on theory. An example of a theoretically appropriate justification for specifying an error covariance may exist when a latent factor (i.e., depression, intelligence, anxiety, etc.) is measured over time with the same scale or parallel form. For instance, participants from two populations take an attitudinal scale at two time points (i.e., pre- and post-intervention) and a CFA model is designed to assess the measurement properties of the scale (i.e., assess if measurement invariance holds). It would be theoretically appropriate and justifiable to allow the error variances to correlate over time for the 
same items at each measurement period. Furthermore, many researchers have investigated how error covariance specification can affect SEM parameters and even argue that in certain situations error covariance specification is advised and should be implemented into the model (Ferron, Dailey, \& Yi, 2002; Grimm \& Widaman, 2010; Kwok, West, \& Green, 2007; Sivo, Fan, \& Witta, 2005).

Given the rational above, strict factorial invariance is the most stringent condition to satisfy and may not be reached in applied research situations. In Schmitt, Pulakos, and Lieblein (1984), the test of $\Theta_{\delta \mathrm{k}}=\Theta_{\delta k^{\prime}}$ was used to indicate invariant reliabilities across groups. However, as described in Millsap (2005) and Vandenburg and Lance (2000) this is only correct if the factor variances are invariant across groups. That is, factor variances and covariances affect the estimate of reliability as well as the uniqueness of an item. Therefore, reliability can be calculated using only error variances when the factor variances are invariant and no covariances exist between the latent factors.

Invariant Factor variances. This is the first test of structural invariance (SI) and is a test of invariant factor variances across groups, $\Phi^{\mathrm{k}}{ }_{\mathrm{j}}=\Phi^{\mathrm{k}_{\mathrm{j}}}$. In other words, it is a hypothesis of whether like factor variances ( $\xi$; diagonal of $\Phi$ ) across groups are the same by placing equality constraints across the groups. Factor variances are a measure of variability on the latent construct and are useful for interpreting the groups' dispersion on a specific construct. Larger variances indicate more variability in the latent factor, whereas, smaller variances indicate less variability (i.e., more consistency) in the latent factor. If the null hypothesis of equal factor variances is rejected then the groups do not have the same amount of dispersion on the latent factor. As mentioned above, factor variances (and covariances) are also important to consider when calculating reliability estimates of the items. 
Invariant Factor covariances. This is the second test of structural invariance and evaluates if the factor covariances are equal across groups, $\Phi^{\mathrm{k}}{ }_{\mathrm{jj}}=\Phi^{\mathrm{k}_{\mathrm{jj}}}$. The null hypothesis for this test is that the factor covariances, for like factors, are equal across groups. Rejection of the hypothesis indicates that the relationships between the latent factors are different across groups. That is, groups can perceive relationships of different magnitude or even direction between the latent constructs. Vandenburg and Lance (2000) purposed that the test of factor covariances leaves little information to be gained between the groups, because if the magnitude and direction of factor covariances are very different, then the test of configural invariance would have also likely been rejected. Furthermore, Vandenburg and Lance (2000) stated that if the test of configural invariance was not rejected but the test of invariant factor covariances was rejected, they would be more inclined to agree with the test of configural invariance.

Invariant Factor means. Finally, the last structural test is the test of equality for latent factor means across groups and is represented as $\kappa_{\mathrm{k}}=\kappa_{\mathrm{k}^{\prime}}$. If the null hypothesis of factor means is rejected then the groups do not share the same mean on the latent construct. However, if testing two groups (e.g., depressed and non-depressed) on a particular measure (e.g., some normed depression test) it may be conceptually meaningful to have different latent factor means, given the appropriate substantive research hypothesis. That is, the two groups should be expected to score differently on the measure, because they have two different levels of depression. If the measure did not differentiate between the depressed and non-depressed individuals then it would be useless. Therefore, the equality test of factor means should be theoretically justified before directly testing invariance of factor means.

In summary, the sequential testing of MI can be evaluated in four general steps. The test of configural invariance needs to be established first. If each group does not fit the same latent 
structure, then further MI testing is unnecessary. The next step is testing metric invariance, which evaluates invariant factor loadings between the groups. The third step is evaluating scalar invariance which determines if the item intercepts (i.e., item means) are invariant between groups, in addition to the factor loadings. Finally, the last step is strict invariance which evaluates if the item residuals (i.e., uniqueness) are invariant by group, in addition to the factor loadings and intercepts. To conclude the discussion of MI, Vandenburg and Lance (2000) provided a few reasons supporting why MI under a CFA framework is typically more advantageous than traditional analytic approaches. First, before conducting traditional tests like analysis of variance (ANOVA) or multivariate analysis of variance (MANOVA), one must ensure MI between the groups. Once it is established that both groups perceive the items the same, it is then appropriate to continue with testing group differences between observed or latent measures. Therefore, the advantage of CFA over traditional analytic approaches is that latent means are disattenuated for measurement error and provide a more accurate representation of true population differences. The last advantage of MI testing is being able to assess partial invariance, which occurs when some but not all parameters are invariant, and is not available in traditional analytic approaches. Given an understanding of MI and the sequential testing process, which involves the use of fit indices, the following section will provide a review of the GOF indices used for the current study along with their associated formulas.

\section{Goodness of Fit Indices Evaluated for the Current Study}

The fit indices evaluated in this study are the Comparative Fit Index (CFI), the root mean square error of approximation (RMSEA), the standardized root mean square residual (SRMR), Gamma hat, and McDonald's (Mc) index. Each index is described along with the associated equation and recommendations for cutoff criteria (i.e., what is considered a good model fit). 
However, before any further discussion on goodness-of-fit (GOF) indices, I provide a brief introduction in structural equation modeling (SEM) and confirmatory factor analysis (CFA). SEM and CFA has become common tools across multiple disciplines and fields for investigating theoretical models to represent how a group of variables are related (Hu \& Bentler, 1999). The first step is to appropriately specify a model using a strong theoretical rationale. Once a particular model is specified, fit indices are used to assess how well the model fits the data. If the model fits the data well, a researcher can conclude that their model is one of the true, plausible models in the population. However, if the fit indices do not indicate adequate fit then the model is rejected. Therefore, a solid understanding of what fit indices represent and their associated equations are essential for researchers who use SEM or CFA.

GOF indices are used to determine how well the model-implied covariance matrix $(\hat{\Sigma})$ equals the sample covariance matrix (S) (Bollen, 1989; Marsh, Hau, \& Grayson, 2005). One of the most commonly used fit indices is the chi-square test statistic $\left(\chi^{2}\right.$, Cheung \& Rensvold, 2002). The $\chi^{2}$ goodness-of-fit test statistic is a measure of exact fit and assesses the discrepancy between the sample and model-implied covariance matrices (Millsap, 2007). Defined below, it is the product of the minimum fitting function and the sample size $(N)$ minus one

$$
T=(N-1) F_{\min }
$$

where $F_{\min }$ is defined as the minimum fitting function

$$
F_{\min }=\log |\Sigma(\theta)|+\operatorname{tr}\left(\mathrm{S} \Sigma^{-1}(\theta)\right)-\log |\mathrm{S}|-(p+q)
$$

where $\Sigma(\theta)$ is the model implied matrix, $\theta$ are the model parameters, $\mathrm{S}$ is the sample covariance matrix, $p$ is the number of endogenous $(\eta)$ observed indicators, and $q$ is the number of exogenous 


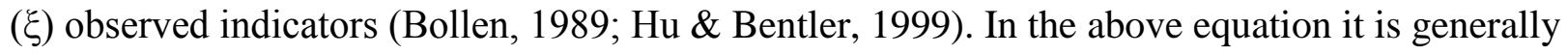
assumed that $\Sigma(\theta)$ and $\mathrm{S}$ are positive definite, meaning nonsingular, otherwise $F_{\min }$ could result in an undefined log of zero (Bollen, 1989). If the sample data perfectly fits the model implied matrix, $\hat{\Sigma}=\mathrm{S}$, substitute $\mathrm{S}$ for $\Sigma(\theta)$ and $F_{\min }$ will then be equal to zero as shown below

$$
F_{\text {min }}=\log |\mathrm{S}|+\operatorname{tr}(\mathrm{I})-\log |\mathrm{S}|-(p+q)
$$

Where $\operatorname{tr}(\mathrm{I})=p+q$ and both of the $\log |\mathrm{S}|$ terms cancel, therefore reproducing a perfect fit (i.e., $F_{\min }$ equal to zero) (Bollen, 1989). Rejecting the $\chi^{2}$ indicates the sample covariance matrix and model implied matrix are not an exact fit (Millsap, 2007). However, theoretical models will not exactly represent the sample covariance matrix. Therefore, other fit indices and methods have been developed and are described below.

When testing sequentially nested models for MI properties (i.e., a more constrained model is subsumed under a less constrained model), researchers may use delta goodness-of-fit ( $\triangle \mathrm{GOFs}$ ) indices to evaluate the fit of competing models or the chi-square difference test (a.k.a LR test). However, if models are non-nested then information criteria (e.g., AIC, BIC, CAIC) can be used to evaluate model fit, where smaller values indicated better fit (Bollen, 1989; Cudeck \& Browne, 1983). The LR test is used to evaluate if two nested models are significantly different by conducting a chi-square difference test between the two models which uses the original chi-square values from both models (Bollen, 1989). However, the LR test has several assumptions that must hold, but are usually violated in practice. The assumptions are (1) the observed variables have no kurtosis (i.e., the best distribution is multinormal), (2) the covariance matrix is analyzed, not the correlation matrix, (3) sufficiently large (> 100) sample size (Anderson \& Gerbing, 1984), and (4) the null hypothesis that the sample and model-implied 
matrices are equal holds exactly (Bollen, 1989). Given the strict assumptions of the LR test, it is understandable why other measures of fit are also used to sequentially test models.

As with the LR test there are concerns with $\triangle \mathrm{GOF}$ indices as well. One issue with most of the commonly used GOF indices is that they are descriptive in nature and do not have known probability distributions. That is, GOF indices can only be used to describe how well a model fits the data and cannot be used inferentially. Therefore, general cutoff values are provided as appropriate criterion as to whether a particular model fits the data or does not fit the data (Cheung \& Rensvold, 2002). Also, there are no known probability distributions (i.e., $p$ values) that can be used to compare nested models (like the LR test) when using alternative GOF indices. Presented below is a summary of each GOF index used for the current study along with their recommended cutoff values for adequate model fit. The cutoff values recommended by Hu and Bentler (1999) result in lower Type II error rates along with an acceptable cost of Type I error rates when using maximum likelihood (ML) as the estimation method.

Comparative Fit Index (CFI). This index is an incremental fit index, which measures the improvement of fit of a hypothesized model over a baseline model, and defined below (Bentler, 1990).

$$
C F I=1-\left\{\frac{\operatorname{MAX}\left(\chi_{t}^{2}-d f_{t}, 0\right)}{\operatorname{MAX}\left(\chi_{n}^{2}-d f_{n}, 0\right)}\right\}
$$

where $\chi_{t}^{2}$ is the chi-square for the tested model, $\chi_{n}^{2}$ is the chi-square for the null model, and $d f_{t}$ and $d f_{n}$ are the degrees of freedom for the tested and null model, respectively. The MAX function is used in the case of a negative value being calculated when subtracting the degrees of freedom from the chi-square value. If a negative value is calculated then the zero is used 
(indicated by the $M A X$ function) to keep the upper-bound of the CFI at one, however, if this occurs it does not mean that the model has a perfect fit (Kline, 2011). The CFI describes the reduction of two nested models (Bentler, 1990). That is, the ratio of the null model to the tested model is subtracted from one. Therefore, if the discrepancy between the two models is small then the value should be close to one. Hu and Bentler (1999) recommend that a cutoff value close to or higher than .95 indicates a good model fit when using the CFI.

Root mean square error of approximation (RMSEA). This index proposed by Steiger and Lind (1980) and Steiger (1989) is an absolute type of fit index that measures error of approximation and is defined as

$$
R M S E A=\sqrt{\frac{\chi^{2}-d f}{d f(N-1)}}
$$

where $\chi^{2}$ is the chi-square for the model, $d f$ is the degrees of freedom, and $N$ is sample size. The RMSEA is adjusted for model parsimony because as the number of degrees of freedom increase (i.e., more parsimonious) the RMSEA value decreases, holding all else constant. If sample size is not held constant, then large increases in sample size will diminish the correction for parsimony (Kline, 2011). Therefore, the RMSEA is scaled as a badness-of-fit index (i.e., values close to zero indicate good model fit). $\mathrm{Hu}$ and Bentler (1999) recommend that a cutoff value less than .06 be used to indicate a good model fit. Moreover, MacCallum, Browne, and Sugawara (1996) indicated that .01, .05, and .08 imply excellent, good, and fair fit, respectively. Another good quality of the RMSEA is that a confidence interval can be computed to assess the accuracy of the point estimate. Generally, if the lower bound is less than .05 or close to zero and the upper bound 
is not more than .08 , the hypothesized model can be supported when using the RMSEA (Kline, 2011).

Standardized root mean square residual (SRMR). The standardized root mean square residual (Jöreskog \& Sörbom, 1988) is an absolute fit index that measures error of approximation and is defined as

$$
S R M R=\sqrt{\frac{2 \sum \sum\left[\left(s_{i j}-\sigma_{i j}\right) /\left(s_{i i} s_{j j}\right)\right]^{2}}{p(p+1)}}
$$

where $s_{i j}$ is the observed covariance of the $i$ th row and the $j$ th column, $\sigma_{i j}$ is the model-implied covariance of the $i$ th row and the $j$ th column, $s_{i i}$ and $s_{j j}$ are the observed standard deviations, and $p$ is the number of observed variables. As evident in the equation above, the SRMR transforms the sample and model implied covariance matrices into correlation matrices (through division of the standard deviations) and assesses the overall discrepancies between the two correlation matrices. Therefore, the SRMR is a measure of the average correlational residuals (Kline, 2011). $\mathrm{Hu}$ and Bentler (1999) recommend that a cutoff value less than .08 indicates a good model fit when using the SRMR. However, Kline (2011) stated that reporting the SRMR summary statistic is not enough. Since the SRMR is the average of the absolute correlation residuals, some values may be much higher than the .08 criteria. Therefore, Kline (2011) suggests that it is better to actually inspect the correlation residuals and their patterns instead of just reporting the SRMR fit statistic.

Gamma hat. Proposed by Steiger (1989) (also called unbiased goodness-of-fit index) is an absolute goodness-of-fit index and is defined as 


$$
\text { Gamma hat }=\frac{p}{\frac{2\left(\chi^{2}-d f\right)}{N-1}+p}
$$

where $p$ is the number of observed variables, $\chi^{2}$ is the model chi-square test statistic, $d f$ is the degrees of freedom for the model, and $N$ is the sample size. Steiger, (1989) stated that Gamma hat "can be thought of as a weighted population coefficient of determination for the multivariate model” (p. 84) and that it is the population equivalent of Jöreskog and Sörbom's GFI. Hu and Bentler (1999) recommend that a cutoff value above .95 indicates a good model fit when using Gamma hat.

McDonald's Index (Mc). Proposed by McDonald (1989) is a centrality-based absolute fit index and defined as:

$$
M c=\exp \left\{\frac{-\left(\chi^{2}-d f\right)}{[2(N-1)]}\right\}
$$

where $\chi^{2}$ is the model chi-square test statistic, $d f$ is the degrees of freedom for the model, and $N$ is the sample size. In McDonald's (1989) seminal article proposing this index, he defined it as a measure of centrality. However, other researchers have used names like McDonald's fit index, noncentrality index, and CENTRA, all to describe Mc (Marsh, Hau, \& Grayson, 2005). McDonald (1989) defined $d$ as a noncentrality measure that can be used to assess "badness-offit." In McDonald's (1989) article the equation for his noncentrality measure is

$$
d=f-(p / n)
$$

where $f=(-2 \log L) / n, p$ is the number of observed variables, $n$ is the sample size, and $\log L$ is defined below as (Bollen, 1989) 


$$
\log L=-\frac{N-1}{2}\left\{\log |\hat{\Sigma}|+\operatorname{tr}\left(\hat{\Sigma}^{-1} S\right)\right\}
$$

However, this study uses his measure of centrality defined above, which is what is currently used when researchers indicate they are using Mc. Hu and Bentler (1999) recommend that a cutoff value higher than .90 indicates a good model fit when using McDonald's index.

Following, is a summary of simulation studies used to investigate the MI properties among different models and research designs. The section will include five articles that used simulation studies to evaluate the sensitivity of selected fit indices to evaluate measurement invariance properties. They will be described in chronological order with the first study conducted in 2002 and the most recent study conducted in 2009.

\section{Previous Research}

Cheung and Rensvold (2002) used a simulation study to examine changes in 20 fit indices $(\triangle \mathrm{GOF})$ when imposing cross-group constraints on a first-order measurement model. The design conditions consisted of 48 different models (replicated 1,000 times for each condition) by varying certain parameters (i.e., number of factors, number of variables, factor loadings, factor variance, factor correlations, and sample size per group). Three fit indices (i.e., $\Delta \mathrm{CFI}, \Delta \mathrm{Gamma}$ hat, and $\Delta \mathrm{Mc}$ ) were recommended because they are independent of model complexity and sample size, in addition to not being correlated with the overall fit measure (i.e., providing redundant information). Cheung and Rensvold (2002) also suggested invariance holds when the critical value is smaller than or equal to -.001 for $\Delta$ Gamma hat, -. .02 for $\Delta \mathrm{Mc}$, and -.01 for $\Delta \mathrm{CFI}$.

French and Finch (2006) further investigated MI properties of first-order models using $\Delta \chi^{2}, \Delta \mathrm{CFI}$, and a combination of $\Delta \chi^{2}$ and $\Delta \mathrm{CFI}$. Their simulation study included varying sample 
size, normality of variables, number of factors, number of variables, and factor loading differences. French and Finch (2006) considered a lack of invariance for $\Delta$ CFI as any value less than -.01, as also recommended by Cheung and Rensvold (2002), and a significant $\Delta \chi^{2}(p<.05)$ for the likelihood ratio test. For the combined condition (i.e., combination of $\Delta \chi^{2}$ and $\Delta \mathrm{CFI}$ ) models were considered non-invariant when both conditions were met. The authors found that the $\Delta \chi^{2}$ (using an alpha of .05) provided good control of Type I error rate and high power for most situations while the observed variables were normally distributed and ML estimation was used. Using an alpha of .01, the $\Delta \chi^{2}$ also has good control of Type I error but, as expected, less power. When comparing the $\Delta \chi^{2}$ and the $\Delta$ CFI test, the $\Delta \chi^{2}$ (using an alpha of .01) had more power than the $\Delta$ CFI test. In addition, when both the $\Delta \chi^{2}$ and $\Delta$ CFI were used together the power was too low and therefore the authors recommend not using both fit indices simultaneously. In summary, French and Finch (2006) stated that $\Delta$ CFI was not as consistent as the $\Delta \chi^{2}$ and with smaller sample size (i.e., $n=300$ ) the Type I error rate of the $\Delta$ CFI drastically increased up to 10 times greater than the nominal alpha rate.

The third study was conducted by Chen (2007) and included two Monte Carlo studies to investigate the sensitivity of $\Delta \mathrm{GOF}$ indices and a lack of $\mathrm{MI}$ in first-order models. In the first study, Chen (2007) investigated the sampling variability of three common fit indices (i.e., SRMR, CFI, and RMSEA), in addition to the two recommended fit indices (i.e., Gamma hat and Mc) by Cheung and Rensvold (2002). Chen (2007) established cutoff points for deciding if invariance holds between two models by examining GOF indices' percentiles and Type I error rates. Chen (2007) generated 500 replications per condition, which varied the number of groups, number of indicators per factor, number of factors, and sample size. Chen (2007) recommends values of $\leq-.005$ to -.010 for CFI, a change of $\geq .010$ to .015 for RMSEA, change of $\leq-.005$ to 
-.008 for Gamma hat, and a change of $\leq-.010$ to -.015 for Mc to indicate invariance holds. Chen's (2007) results for CFI, RMSEA, and Mc are consistent with Cheung and Rensvold's (2002) results. However, Chen (2007) recommends different values for SRMR based on the different levels of invariance tests (i.e., loading, intercept, and residual variance). For SRMR a change of $\geq .025$ to .030 is recommend for invariance tests of factor loadings and a change of $\geq$ .005 to .010 is recommend for intercept level and residual variance tests. The second part of Chen's (2007) study investigated the $\triangle$ GOFs at three levels of non-invariance (i.e., factor loadings, intercepts, and residual variances) in varied conditions: number of groups, number of indicators, number of factors, proportion of invariance (i.e., proportion of factor loadings differing across groups), pattern of invariance (i.e., uniform or mixed - all loadings higher in one group or varying by group), and ratio of sample size (i.e., ratio of the size of the two groups). One of the seminal findings from the second study occurred when testing invariance at the factor loadings and intercepts level. The interaction between the magnitude of loadings and pattern of invariance affected the $\triangle \mathrm{GOF}$ indices making the relationship non-monotonic. That is, when lack of invariance was largest between the groups, changes in fit indices were the smallest. When lack of invariance was moderate between the groups, changes in fit indices were the largest. Therefore, Chen (2007) concludes by stating that MI testing is a very complex topic, as shown in her study, and several factors (e.g., pattern of invariance, proportion of invariance, etc.) all contribute to the sensitivity of the fit indices for a specified first-order model.

Meade, Johnson, and Braddy (2008) conducted a simulation study to investigate the limitations of Cheung and Rensvold's (2002) study. The first limitation of Cheung and Rensvold's (2002) study was that they only simulated data with perfect invariance and did not investigate models with a lack of invariance. The second major limitation is that they grouped 
$\triangle \mathrm{GOF}$ indices across a variety of MI tests (i.e., factor loadings, item intercepts, etc.) to provide overall average cutoff values. Therefore, Meade et al. (2008) designed their study to investigate the aforementioned limitations of Cheung and Rensvold's (2002) study. First, Meade et al. (2008) investigated several fit indices when models were not perfectly invariant (i.e., a lack of invariance). Second, they examined cutoff values for $\Delta \mathrm{GOF}$ indices across different types of MI tests (e.g., metric and scalar invariance). Meade et al.'s (2008) study was also based on the firstorder model.

There were three phases in Meade et al.'s (2008) study. The first was to simulate data for two groups that varied the level of invariance. The second phase determined cutoff values for varying conditions (e.g., number of factors, number of indicators per factor, sample size, percentages of items considered invariant, and the magnitude of invariance) under the null hypothesis of invariance. The third phase examined power and Type I error rates. Meade et al. (2008) found that all $\Delta$ GOFs were sensitive enough to detect a sizable lack of configural invariance, with $\Delta \mathrm{Mc}$ being the best index of detection. Several indices (e.g., $\Delta \mathrm{CFI}, \Delta \mathrm{Mc}, \Delta \mathrm{IFI}$, $\Delta \mathrm{RNI}, \Delta$ Gamma hat, and $\Delta \mathrm{RMSEA}$ ) were sensitive to a lack of invariance while being unaffected by sample size, number of items, number of factors, and interactions among those variables. In addition, all of the indices tested performed better than the traditional likelihood ratio test.

The results of the Meade et al.'s (2008) study concur with Cheung and Rensvold's (2002) study in that $\triangle \mathrm{CFI}$ and $\Delta \mathrm{Mc}$ are the best indices to report because they are non-redundant with other $\triangle \mathrm{GOF}$ indices. In addition, $\Delta \mathrm{CFI}$ and $\Delta \mathrm{Mc}$ are less sensitive to sample size than chisquare, but more sensitive to a lack of invariance. However, Meade et al. (2008) recommended 
different cutoff values for $\Delta \mathrm{CFI}$ and $\Delta \mathrm{Mc}$ than Cheung and Rensvold (2002). Meade et al. (2008) recommended invariance cutoff values as changes less than .002 for $\Delta \mathrm{CFI}$ and .008 for $\Delta \mathrm{Mc}$.

Recently, Fan and Sivo (2009) investigated $\Delta$ GOF indices (e.g., $\Delta$ RMSEA, $\Delta$ NFI, $\Delta$ TLI, $\Delta \mathrm{CFI}, \Delta \mathrm{Gamma}$ hat, $\Delta \mathrm{McDonald}$ 's, etc.) for mean structure analyses (i.e., population latent means) to test MI properties. Using a Monte Carlo study, they extended the previous work by Cheung and Rensvold (2002) who only investigated MI properties in terms of the covariance structure. Fan and Sivo (2009) tested three measurement models where they varied the number of latent factors and number of observed indicators per factor. However, Fan and Sivo (2009) were only interested in mean structure analysis and allowed the factor loadings, variances, covariances, and residual variances to be the same for both of the simulated populations. The mean structure of the model differed by each group in terms of effect sizes (i.e., standardized mean differences) from null (i.e., zero) to a large effect size of .80. Fan and Sivo (2009) indicated that the $\triangle \mathrm{GOF}$ indices are sensitive to model complexity (i.e., number of indicators and latent factors) and are not useful for conducting mean structure invariance analysis. In other words, the power of the $\triangle \mathrm{GOF}$ indices for detecting population mean differences decreases as the models become more complex (i.e., more factors and indicators per factor) which is opposite of what is expected (Fan \& Sivo, 2009). Therefore, Fan and Sivo (2009) recommend that $\Delta \mathrm{GOF}$ indices are not appropriate for mean structure invariance testing.

All of the previous studies contribute to the field of MI. However, to my knowledge no one to date has investigated the MI properties of the bifactor model, which is a higher-order model. The bifactor model was first proposed by Holzinger and Swineford (1937) in Psychometrika when the authors summarized the Preliminary Reports on the SpearmanHolzinger Unitary Trait Study (1930-1936) using the results of a small sample who completed 14 
tests. The bifactor model is simply an extension of Spearman's two-factor pattern, which consists of a general factor that loads on all items and additional uncorrelated unique factors. In other words, the bifactor model has a general factor (that loads on each item) and specific uncorrelated group factors, which contain covariance among a group of items over and above the general factor (Holzinger \& Swineford, 1937).

The bifactor model is becoming increasingly more popular in applied research and can be used within the areas of intelligence research and method effects (Brown, Finney, \& France, 2011; Chen, West, \& Sousa, 2006; DiStefano \& Motl, 2006; Gustafsson \& Balke, 1993; Jennrich \& Bentler, 2012; Luo, Petrill, \& Thompson, 1994). Therefore, it is foreseeable that the MI properties of higher-order models and the sensitivity of $\Delta \mathrm{GOF}$ indices to detect non-invariance in higher-order models will need to be investigated. As found in Chen's (2007) study of $\Delta \mathrm{GOF}$ indices for the first-order model, the interaction between the magnitude of factor loadings differences and the pattern of invariance can yield complex results. Using Chen's (2007) study as a foundation, it is likely that the higher-order models will be more multifaceted. Therefore, simulations studies are needed to evaluate the sensitivity of $\Delta \mathrm{GOF}$ indices to detect noninvariance in higher-order models. Given that the MI properties of the bifactor model have not been evaluated, the sensitivity of several fit indices (i.e., $\triangle \mathrm{CFI}, \triangle \mathrm{RMSEA}, \Delta \mathrm{SRMR}, \Delta \mathrm{Gamma}$ hat, and $\Delta$ McDonald's) will be investigated using this model. 


\section{METHOD}

The current study was designed to investigate some of the unresolved issues from previous research and help to fill a gap in the literature. To accomplish this, a CFA model was used to evaluate the measurement invariance properties of the bifactor model while manipulating several conditions to assess what effects the non-invariant parameters have on the GOF indices. The purpose of this study is measurement invariance, which assumes a theoretical model is hypothesized a priori. Therefore, the current study focuses on investigating only bifactor models and is not concerned with misspecification (i.e., unidimensional or multidimensional). The study investigated two bifactor models using twenty items with varying conditions.

\section{Bifactor Models}

The first model was classified as a simple bifactor model, consisting of twenty normally distributed items and three latent factors (i.e., one general and two specific). The general factor loaded on all twenty items and the first specific factor loaded on the first ten items whereas, the second specific factor loaded on the last ten items (see Figure 2). The second model was classified as a complex bifactor model, consisting of twenty normally distributed items and five latent factors (i.e., one general and four specific). Again, the general factor loaded on all twenty items and the first specific factor loaded on the first five items, the second specific factor on the second set of five items, the third specific factor on the third set of five items, and finally the fourth specific factor loaded on the last set of five items (see Figure 3). These types of models are typically used for attitudinal scales and can be used to examine method effects as well (DiStefano \& Motl, 2006). The models were chosen to yield the most consistent results. In other words, as the bifactor model becomes more complex the findings may become more erratic, leading to more difficult or inconsistent conclusions. 


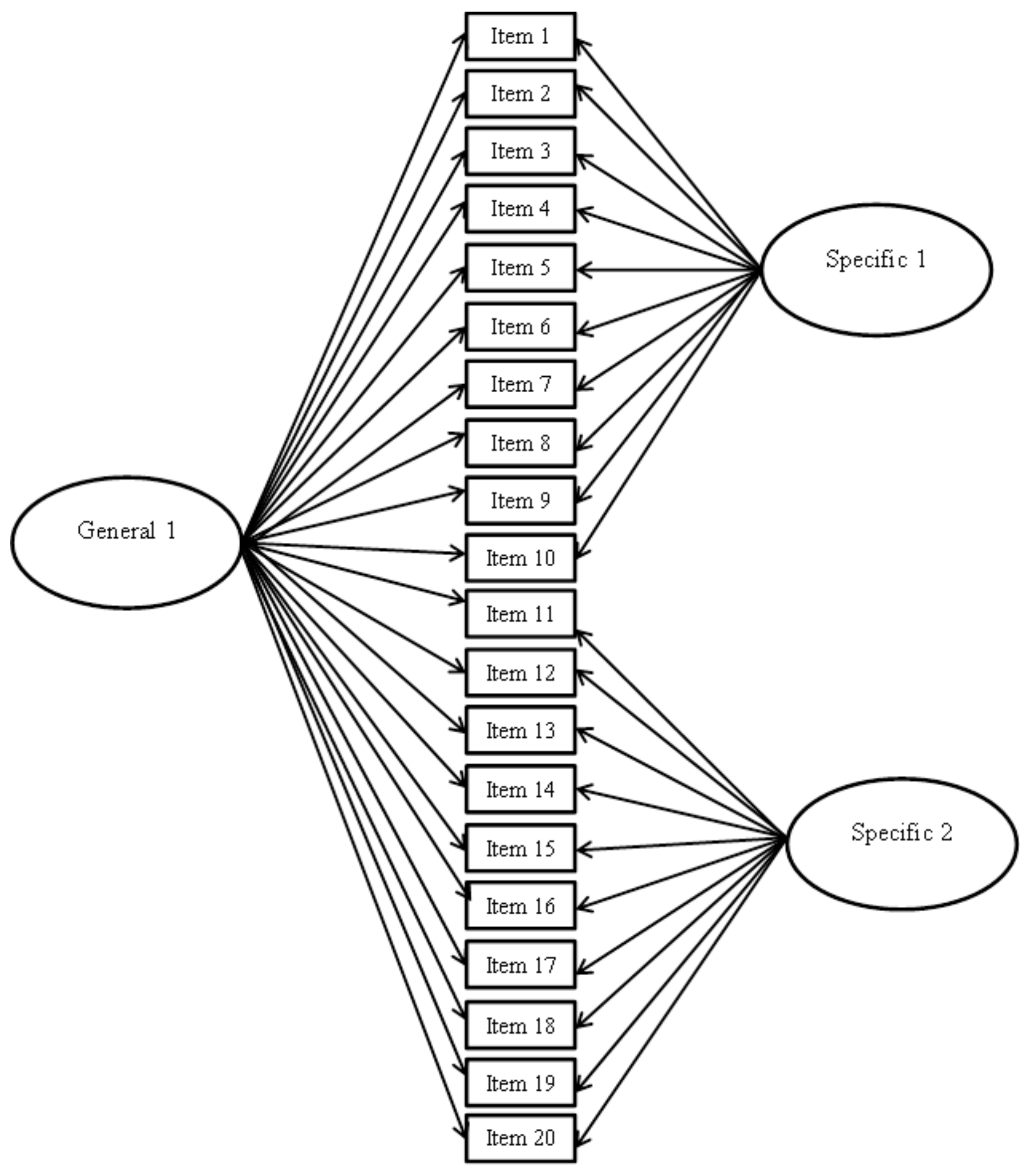

Figure 2. Simple model used for simulation, one general and two specific factors. 


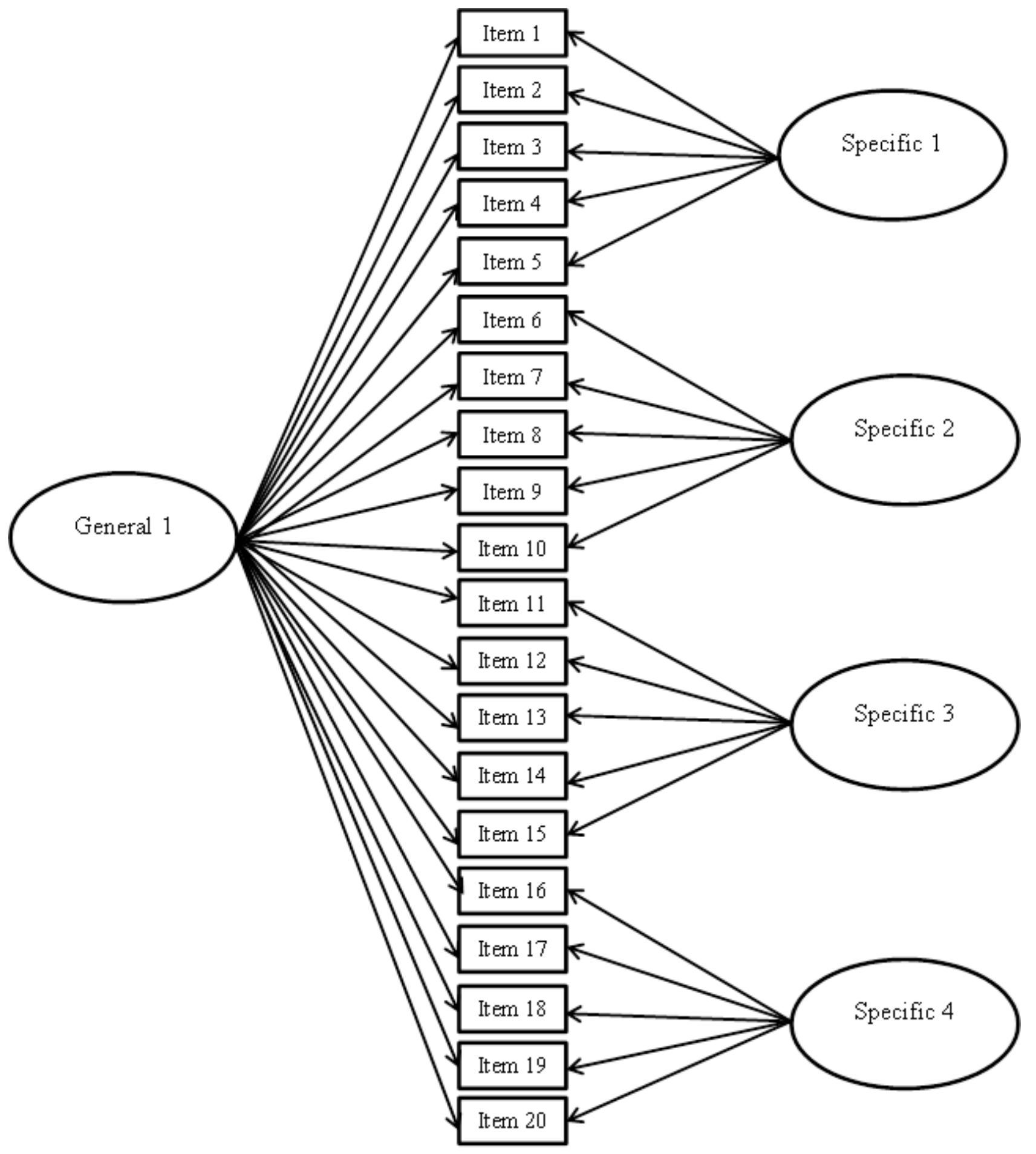

Figure 3. Complex model used for simulation, one general and four specific factors 
The following sections provide a summary of the parameters controlled followed by subsections detailing each condition. The set of conditions were applied to both the simple and complex bifactor models. Also presented in the methods section were the equations used to generate the data along with algebraic extensions from the traditional one factor CFA model to the more complex bifactor model.

\section{Simulation Conditions}

Five major parameters were manipulated in this study: 1) sample size per group, 2) magnitude of factor loadings differences, 3) factors with non-invariant loadings, 4) percentage of specific factors with non-invariant items, and 5) percentage of non-invariant items.

Sample size. The minimum sample size was set to be large enough so the bifactor models can converge, and sample sizes for the two groups were selected to be equal. Therefore, the sample sizes per group were 400, 800, and 1200 yielding total sample sizes of 800, 1600, and 2400 , respectively.

Magnitude of factor loading differences. The small and large magnitude of factor loading differences between the groups were .2 and .4 , respectively. A factor loading difference greater than .4 may not be conceptually meaningful to simulate. That is, a factor loading difference greater than .4 is strong evidence that the item is non-invariant between the two groups and further MI testing for that item may be problematic. The factor loadings for group 1 served as the reference group to compute the magnitude of factor loading differences for group 2. Group 1 was the group with the larger factor loadings. The factor loadings for group 2 were set by subtracting the magnitude differences of .2 or .4 from group 1 . All of the factor loadings for the first group were set at .7 for both the specific and general factors. The factor loadings for the 
second group were set at .7 when invariance existed. However, when the loadings were noninvariant across groups, the factor loadings were .5 or .3 for small and large magnitude differences, respectively.

Factors with non-invariant loadings. This condition specifies the location of the noninvariant loadings for both the general and specific factors and was generated to be most practical for applied researchers. That is, conceptually non-invariant loadings could exist on only the specific factors or both the general and specific factor (for the same item). However, it is unlikely that the general factor would be non-invariant and the specific factor invariant, because the specific factor is the covariance among a group of items over and above the general factor (Holzinger \& Swineford, 1937). Therefore, this condition was not simulated. To summarize, for both the simple and complex models one condition had non-invariant loadings on only the specific factor and the other condition had the non-invariant loadings on the specific factor and the associated loadings on the general factor for certain items.

Percentage of specific factors with non-invariant items. This condition specifies whether the non-invariant items occur on all of the specific factors (i.e., 100\%) or only half of the specific factors (i.e., 50\%) for both the simple and complex models. For example, in the simple model, $100 \%$ indicates that non-invariant items were located on both specific factors and the $50 \%$ condition indicates that non-invariant items were located on only one of the specific factors. The same logic is extended to the complex model where $100 \%$ indicates the noninvariant items were on all four of the specific factors and 50\% indicates that the non-invariant items were on only two of the four specific factors. 
Percentage of non-invariant items. This condition specifies the percentage of noninvariance for the twenty items. The two conditions were $20 \%$ (i.e., $4 / 20$ ) and $40 \%$ (i.e., $8 / 20$ ) of the total items were non-invariant.

Table 1 summarizes the simulation conditions used for the study. There were a total of 48 conditions (i.e., 3x2x2x2x2) for each bifactor model (i.e., simple and complex) replicated 1000 times for a total of 96,000 data sets.

Table 1

Summary of Conditions for Simulation Study

\begin{tabular}{ll} 
Parameters Controlled & Condition Options \\
\hline$n$ (per group) & $400,800,1200$ \\
Magnitude of Factor Loading Differences & .2 or .4 \\
Factors with Non-Invariant Items & Specific or Both General \& Specific \\
Percentage of Specific Factors Non-Invariant & $50 \%$ or $100 \%$ \\
Percentage of Non-Invariant Items & $20 \%$ or $40 \%$ \\
\hline
\end{tabular}

\section{Fit Indices and Estimation}

Based on previous researchers' recommendations (Cheung \& Rensold, 2002; French \& Finch, 2006; Chen, 2007; Meade, Johnson, \& Braddy, 2008; Fan \& Sivo, 2009), five fit indices are used in this study (i.e., CFI, RMSEA, SRMR, Gamma hat, and Mc). The simulated data will be normally distributed and estimated using maximum likelihood (ML). ML is one of the most commonly used estimation techniques for normally distributed data and all model parameters are simultaneously calculated at once (Kline, 2011). 


\section{Equations to Generate Data}

All of the data generation, estimation, and analyses will be conducted using EQS 6.1 and SAS 9.3. The equations used to generate the data are derived from the factor loading matrix $(\Lambda)$. As typical with most simulation research, the factor variance was set to one for model identification instead of constraining a factor loading to be one from each group for each latent factor (Bollen, 1989; Millsap \& Kwok, 2004; Yoon \& Millsap, 2007). The factor loadings were specified for each group (based on the conditions above) and the error and covariance matrices were calculated from the lambda matrix. The general forms of the CFA and SEM equations were

provided in Chapter 2. The following sections provide specific examples of the equations used to simulate the models. To aid in the discussion of the bifactor equations assume a factor loading matrix like the one below, where all twenty items load on the general factor, the first ten items load on the first specific factor, and the last ten items load on the second specific factor (i.e., the simple bifactor model used). 


$$
\Lambda_{x}=\left[\begin{array}{ccc}
\lambda_{10} & \lambda_{11} & 0 \\
\lambda_{20} & \lambda_{21} & 0 \\
\lambda_{30} & \lambda_{31} & 0 \\
\lambda_{40} & \lambda_{41} & 0 \\
\lambda_{50} & \lambda_{51} & 0 \\
\lambda_{60} & \lambda_{61} & 0 \\
\lambda_{70} & \lambda_{71} & 0 \\
\lambda_{80} & \lambda_{81} & 0 \\
\lambda_{90} & \lambda_{91} & 0 \\
\lambda_{100} & \lambda_{101} & 0 \\
\lambda_{110} & 0 & \lambda_{112} \\
\lambda_{120} & 0 & \lambda_{122} \\
\lambda_{130} & 0 & \lambda_{132} \\
\lambda_{140} & 0 & \lambda_{142} \\
\lambda_{150} & 0 & \lambda_{152} \\
\lambda_{160} & 0 & \lambda_{162} \\
\lambda_{170} & 0 & \lambda_{172} \\
\lambda_{180} & 0 & \lambda_{182} \\
\lambda_{190} & 0 & \lambda_{192} \\
\lambda_{200} & 0 & \lambda_{202}
\end{array}\right]
$$

Traditional CFA equations. Before introducing the bifactor equations, example equations of a traditional one factor CFA model are presented. The following section will then extend the examples provided in the traditional CFA equations section to the more complex bifactor equations. To calculate the variance-covariance matrix, the factor loadings $(\lambda)$, factor variance $(\Phi)$, and the error variances $(\delta)$ are all necessary components. For example, in a traditional, single factor CFA model the variance of the first item is defined as

$$
\lambda_{11}^{2} \phi_{11}+\operatorname{VAR}\left(\delta_{1}\right)
$$

where $\lambda_{11}^{2}$ is the factor loading for the first item on the latent factor, $\phi_{11}$ is the corresponding factor variance, and $\operatorname{VAR}\left(\delta_{1}\right)$ is the error variance for the first item (defined below in equation 
3.4) (Bollen, 1989). The covariance between the first two items, represented as $\operatorname{COV}\left(\mathrm{X}_{1}, \mathrm{X}_{2}\right)$, can be decomposed into the product of the factor loading and the factor score plus the error term for both items defined as (Bollen, 1989).

$$
x_{1}=\lambda_{1} \xi_{1}+\delta_{1} \quad \text { and } \quad x_{2}=\lambda_{2} \xi_{1}+\delta_{2}
$$

The terms from each item can be factored and appropriate terms canceled (i.e., $\operatorname{COV}\left(\xi_{1}, \delta_{1}\right)=$ $\left.\operatorname{COV}\left(\xi_{1}, \delta_{2}\right)=\operatorname{COV}\left(\delta_{1}, \delta_{2}\right)=0\right)$ to define the covariance between the first two items as

$$
\lambda_{11} \lambda_{21} \phi_{11}
$$

where $\lambda_{11}$ is the factor loading of the first item on the factor, $\lambda_{21}$ is the factor loading of the second item on the factor, and $\phi_{11}$ is the latent factor variance. The error variance (theta-delta, $\left.\Theta_{\delta}\right)$ is defined below as

$$
1-\lambda_{11}^{2}
$$

where $\lambda_{11}^{2}$ is the factor loading for the first item on the factor. Therefore, it is clear that the remaining variation not accounted for by the factor loading is due to error, where strong (i.e., close to |1|) factor loadings have a stronger relationship with the latent factor resulting in less error and weak (i.e., close to $|0|$ ) factor loadings have more error. The next set of equations is presented to show how the traditional CFA model extends to the bifactor model.

Bifactor equations. The bifactor model items' variance and covariance terms change to include not only the specific factor loadings, but the general factor loadings as well. For example, the variance for the first item of the bifactor model becomes

$$
\lambda_{10}^{2} \phi_{00}+\lambda_{11}^{2} \phi_{11}+\lambda_{12}^{2} \phi_{22}+\operatorname{VAR}\left(\delta_{1}\right)
$$


where $\lambda_{10}^{2}$ is the factor loading for the first item on the general factor, $\phi_{00}$ is the general factor variance, $\lambda_{11}^{2}$ is the factor loading for the first item on the first specific factor, $\phi_{11}$ is the variance for the first specific factor, $\lambda_{12}^{2}$ is the factor loading for the first item on the second specific factor, $\phi_{22}$ is the variance for the second specific factor, and $\operatorname{VAR}\left(\delta_{1}\right)$ is the remaining error variance for the first item (defined in equation 3.8 for the bifactor model). Next, the item covariances for the first two items of the bifactor model can be decomposed into the following terms for each item.

$$
x_{1}=\lambda_{10} \xi_{0}+\lambda_{11} \xi_{1}+\lambda_{12} \xi_{2}+\delta_{1} \quad \text { and } \quad x_{2}=\lambda_{20} \xi_{0}+\lambda_{21} \xi_{1}+\lambda_{22} \xi_{2}+\delta_{2}
$$

However, note that the first two items do not load on the second specific factor $\left(\xi_{2}\right)$ therefore the appropriate terms will be canceled due to the zero factor loadings, but for demonstration purposes the equations are presented using all three latent factors. After factoring and canceling the appropriate terms the covariance between the first two items now becomes

$$
\lambda_{10} \lambda_{20} \phi_{00}+\lambda_{11} \lambda_{21} \phi_{11}+\lambda_{12} \lambda_{22} \phi_{22}
$$

where $\lambda_{10}$ is the factor loading for the first item on the general factor, $\lambda_{20}$ is the factor loading for the second item on the general factor, $\phi_{00}$ is the factor variance of the general latent factor, $\lambda_{11}$ is the factor loading for the first item on the first specific factor, $\lambda_{21}$ is the factor loading for the second item on the first specific factor, $\phi_{11}$ is the factor variance of the first specific latent factor, $\lambda_{12}$ is the factor loading for the first item on the second specific factor (equal to zero), $\lambda_{22}$ is the factor loading for the second item on the second specific factor (equal to zero), and 
$\phi_{22}$ is the factor variance of the second specific latent factor. For the bifactor model, the error term for the first item is defined as

$$
1-\left(\lambda_{10}^{2}+\lambda_{11}^{2}+\lambda_{12}^{2}\right)
$$

where $\lambda_{10}^{2}$ is the factor loading for the first item on the general factor, $\lambda_{11}^{2}$ is the factor loading for the first item on the first specific factor, and $\lambda_{12}^{2}$ is the factor loading for the first item on the second specific factor (equal to zero). The equations presented above will be used to generate the data for the bifactor model and assess the sensitivity of each $\triangle \mathrm{GOF}$ index under investigation.

\section{Analyses}

To evaluate the sensitivity of each fit index, the Type I error rate and power was calculated for metric level invariance and guidelines for establishing invariance were provided. The Type I error rate is the rejection rate for the invariant models, whereas, power is the rejection rate for the non-invariant models. To evaluate the influence of the parameters controlled, a variance component analysis was conducted to evaluate the proportion of variance accounted for in the $\triangle \mathrm{GOF}$ indices by each condition (Harwell, 1997; Harwell, Rubinstein, Hayes, \& Olds, 1992; Hoaglin \& Andrews 1975). Omega-squared was used because it is an estimate of the proportion of variance accounted for in the population and should be used as opposed to eta-squared which is an estimate of the proportion of variance accounted for in a sample (Kirk, 1995).

\section{Arkansas Benchmark Model}

To conclude the study, the bifactor model was applied to the Arkansas Benchmark Exam (ABE) data to evaluate the MI properties of the Arkansas' high-stakes exam. The ABE is an end- 
of-course math and literacy exam administered in grades 3 through 8 in Arkansas and is used for accountability purposes and Adequate Yearly Progress (AYP) calculations. The model consisted of 25 items and six latent factors, which included one general math factor and five specific math strands (i.e., Numbers and Operations with 4 items, Algebra with 5 items, Geometry with 7 items, Measurement with 4 items, and Data Analysis and Probability with 5 items). The measurement invariance properties of the third grade math test were evaluated by gender $(18,678$ boys \& 17,713 girls). First, the test of configural invariance was conducted followed by the equality test of factor loadings (i.e., metric invariance). The chi-square difference test (a.k.a. LR test) was calculated by subtracting the constrained factor loadings (i.e., metric invariance) model from the configural invariance model by group (males and females fitted separately) and used to evaluate if the constrained model fits the data worse than the unconstrained model. The LR test was used to evaluate if a significant chi-square difference test is associated with $\Delta \mathrm{GOF}$ values beyond the recommended cutoff criteria. 


\section{RESULTS}

In this chapter a detailed summary of the analyses conducted are provided. The first section provides the simulation cutoff values used for the bifactor model, followed by Type I error and power rates. The next section describes the proportion of variance accounted for in each $\Delta \mathrm{GOF}$ by condition. Finally, the Arkansas Benchmark Examination (ABE) results will be presented last. A summary of the fit indices for each model are provided along with a brief discussion. Each of the models converged normally, setting the maximum iterations in EQS 6.1 equal to 500 and any models with conditions codes were not used.

\section{Establishing Cutoff Criteria}

The simulation cutoff criteria have to be established before the Type I error and power rates can be determined. The simulation cutoff values were determined by examining the $95^{\text {th }} / 5^{\text {th }}$ percentiles of both the baseline models and non-invariant models. Presented in Tables 2-11 are the number of models used to calculate the $\triangle$ GOFs (i.e., $n$ ), along with the mean, standard deviation, and $95^{\text {th }} / 5^{\text {th }}$ percentile (depending on which fit index is presented) for each of the 96 $\Delta$ GOF conditions (with no condition codes). For example, Table 2 presents $\Delta$ Gamma's descriptive statistics for the complex model and the 48 simulated conditions, whereas Table 3 presents the same descriptive statistics of $\Delta$ Gamma for the simple model and the 48 associated conditions. The remaining eight tables (i.e., Tables 4-11) follow the same format for $\Delta \mathrm{Mc}, \Delta \mathrm{CFI}$, $\triangle \mathrm{SRMR}$, and $\triangle \mathrm{RMSEA}$, respectively. The number of non-invariant models without any condition codes are presented in the $n$ column (column left of $M$ ) in Tables 2-11. That is, $n$ is the total number of replications (out of 1000) that did not have condition codes in either the constrained or unconstrained models and were used to calculate the $\Delta \mathrm{GOFs}$, whereas, $n_{\text {model }}$ is the sample sized used for that model. The negative $\Delta \mathrm{GOF}$ values indicate a lack of fit for indices 
where higher values indicate better model fit (i.e., Gamma Hat, McDonald's, and CFI). Positive $\Delta \mathrm{GOF}$ values indicate a lack of fit for indices where lower values are indicative of better model fit (i.e., SRMR and RMSEA). 
Table 2

\begin{tabular}{|c|c|c|c|c|c|c|c|c|}
\hline Loading & $n_{\text {Model }}$ & Location & Specific $\%$ & Total items $\%$ & $n$ & $M$ & $S D$ & $5^{\text {th }}$ Percentile \\
\hline \multirow[t]{24}{*}{.2} & \multirow[t]{8}{*}{400} & \multirow[t]{4}{*}{ SPEC \& GEN } & \multirow[t]{2}{*}{50} & 20 & 1000 & -0.0487 & 0.0037 & -0.0549 \\
\hline & & & & 40 & 676 & -0.0493 & 0.0053 & -0.0579 \\
\hline & & & \multirow[t]{2}{*}{100} & 20 & 1000 & -0.0494 & 0.0038 & -0.0555 \\
\hline & & & & 40 & 1000 & -0.0729 & 0.0046 & -0.0803 \\
\hline & & \multirow[t]{4}{*}{ SPEC } & \multirow[t]{2}{*}{50} & 20 & 1000 & -0.0413 & 0.0040 & -0.0480 \\
\hline & & & & 40 & 855 & -0.0360 & 0.0054 & -0.0452 \\
\hline & & & \multirow[t]{2}{*}{100} & 20 & 1000 & -0.0422 & 0.0040 & -0.0485 \\
\hline & & & & 40 & 1000 & -0.0591 & 0.0049 & -0.0670 \\
\hline & \multirow[t]{8}{*}{800} & \multirow[t]{4}{*}{ SPEC \& GEN } & \multirow[t]{2}{*}{50} & 20 & 1000 & -0.0489 & 0.0026 & -0.0535 \\
\hline & & & & 40 & 816 & -0.0497 & 0.0030 & -0.0546 \\
\hline & & & \multirow[t]{2}{*}{100} & 20 & 1000 & -0.0496 & 0.0025 & -0.0537 \\
\hline & & & & 40 & 1000 & -0.0730 & 0.0032 & -0.0783 \\
\hline & & \multirow[t]{4}{*}{ SPEC } & \multirow[t]{2}{*}{50} & 20 & 1000 & -0.0415 & 0.0028 & -0.0464 \\
\hline & & & & 40 & 962 & -0.0362 & 0.0032 & -0.0418 \\
\hline & & & \multirow[t]{2}{*}{100} & 20 & 1000 & -0.0426 & 0.0027 & -0.0471 \\
\hline & & & & 40 & 1000 & -0.0595 & 0.0033 & -0.0651 \\
\hline & \multirow[t]{8}{*}{1200} & \multirow[t]{4}{*}{ SPEC \& GEN } & \multirow[t]{2}{*}{50} & 20 & 1000 & -0.0491 & 0.0020 & -0.0524 \\
\hline & & & & 40 & 886 & -0.0499 & 0.0023 & -0.0535 \\
\hline & & & \multirow[t]{2}{*}{100} & 20 & 1000 & -0.0496 & 0.0021 & -0.0530 \\
\hline & & & & 40 & 1000 & -0.0731 & 0.0025 & -0.0771 \\
\hline & & \multirow[t]{4}{*}{ SPEC } & 50 & 20 & 1000 & -0.0416 & 0.0021 & -0.0453 \\
\hline & & & & 40 & 992 & -0.0363 & 0.0024 & -0.0404 \\
\hline & & & 100 & 20 & 1000 & -0.0426 & 0.0022 & -0.0462 \\
\hline & & & & 40 & 1000 & -0.0594 & 0.0027 & -0.0636 \\
\hline .4 & 400 & SPEC \& GEN & 50 & 20 & 1000 & -0.0801 & 0.0047 & -0.0883 \\
\hline & & & & 40 & 348 & -0.0701 & 0.0056 & -0.0800 \\
\hline & & & 100 & 20 & 1000 & -0.0808 & 0.0045 & -0.0883 \\
\hline & & & & 40 & 1000 & -0.1308 & 0.0059 & -0.1400 \\
\hline & & SPEC & 50 & 20 & 1000 & -0.0659 & 0.0052 & -0.0745 \\
\hline & & & & 40 & 342 & -0.0386 & 0.0056 & -0.0473 \\
\hline & & & 100 & 20 & 1000 & -0.0670 & 0.0050 & -0.0750 \\
\hline & & & & 40 & 1000 & -0.1073 & 0.0063 & -0.1176 \\
\hline & 800 & SPEC \& GEN & 50 & 20 & 1000 & -0.0802 & 0.0033 & -0.0858 \\
\hline & & & & 40 & 374 & -0.0703 & 0.0033 & -0.0754 \\
\hline & & & 100 & 20 & 1000 & -0.0811 & 0.0030 & -0.0860 \\
\hline & & & & 40 & 1000 & -0.1308 & 0.0042 & -0.1375 \\
\hline & & SPEC & 50 & 20 & 1000 & -0.0663 & 0.0036 & -0.0724 \\
\hline & & & & 40 & 423 & -0.0385 & 0.0034 & -0.0437 \\
\hline & & & 100 & 20 & 1000 & -0.0676 & 0.0033 & -0.0731 \\
\hline & & & & 40 & 1000 & -0.1078 & 0.0043 & -0.1152 \\
\hline & 1200 & SPEC \& GEN & 50 & 20 & 1000 & -0.0805 & 0.0026 & -0.0849 \\
\hline & & & & 40 & 422 & -0.0706 & 0.0027 & -0.0750 \\
\hline & & & 100 & 20 & 1000 & -0.0811 & 0.0026 & -0.0852 \\
\hline & & & & 40 & 1000 & -0.1310 & 0.0033 & -0.1362 \\
\hline & & SPEC & 50 & 20 & 1000 & -0.0664 & 0.0028 & -0.0711 \\
\hline & & & & 40 & 462 & -0.0385 & 0.0025 & -0.0428 \\
\hline & & & 100 & 20 & 1000 & -0.0676 & 0.0027 & -0.0722 \\
\hline & & & & 40 & 1000 & -0.1078 & 0.0035 & -0.1132 \\
\hline
\end{tabular}


Table 3

\begin{tabular}{|c|c|c|c|c|c|c|c|c|}
\hline Loading & $n_{\text {Model }}$ & Location & Specific $\%$ & Total items $\%$ & $n$ & $M$ & $S D$ & $5^{\text {th }}$ Percentile \\
\hline \multirow[t]{24}{*}{.2} & \multirow[t]{8}{*}{400} & \multirow[t]{4}{*}{ SPEC \& GEN } & \multirow[t]{2}{*}{50} & 20 & 707 & -0.0321 & 0.0061 & -0.0427 \\
\hline & & & & 40 & 694 & -0.0510 & 0.0082 & -0.0644 \\
\hline & & & \multirow[t]{2}{*}{100} & 20 & 554 & -0.0318 & 0.0054 & -0.0414 \\
\hline & & & & 40 & 686 & -0.0544 & 0.0064 & -0.0647 \\
\hline & & \multirow[t]{4}{*}{ SPEC } & \multirow[t]{2}{*}{50} & 20 & 845 & -0.0234 & 0.0055 & -0.0322 \\
\hline & & & & 40 & 888 & -0.0325 & 0.0066 & -0.0433 \\
\hline & & & \multirow[t]{2}{*}{100} & 20 & 844 & -0.0240 & 0.0055 & -0.0334 \\
\hline & & & & 40 & 899 & -0.0402 & 0.0062 & -0.0507 \\
\hline & \multirow[t]{8}{*}{800} & \multirow[t]{4}{*}{ SPEC \& GEN } & \multirow[t]{2}{*}{50} & 20 & 766 & -0.0311 & 0.0042 & -0.0376 \\
\hline & & & & 40 & 749 & -0.0501 & 0.0063 & -0.0622 \\
\hline & & & \multirow[t]{2}{*}{100} & 20 & 636 & -0.0307 & 0.0035 & -0.0360 \\
\hline & & & & 40 & 746 & -0.0532 & 0.0039 & -0.0594 \\
\hline & & \multirow[t]{4}{*}{ SPEC } & \multirow[t]{2}{*}{50} & 20 & 903 & -0.0224 & 0.0031 & -0.0275 \\
\hline & & & & 40 & 917 & -0.0316 & 0.0046 & -0.0400 \\
\hline & & & \multirow[t]{2}{*}{100} & 20 & 897 & -0.0232 & 0.0030 & -0.0281 \\
\hline & & & & 40 & 915 & -0.0393 & 0.0036 & -0.0454 \\
\hline & \multirow[t]{8}{*}{1200} & \multirow[t]{4}{*}{ SPEC \& GEN } & \multirow[t]{2}{*}{50} & 20 & 780 & -0.0307 & 0.0034 & -0.0361 \\
\hline & & & & 40 & 777 & -0.0498 & 0.0051 & -0.0597 \\
\hline & & & \multirow[t]{2}{*}{100} & 20 & 694 & -0.0305 & 0.0026 & -0.0347 \\
\hline & & & & 40 & 782 & -0.0531 & 0.0033 & -0.0582 \\
\hline & & \multirow[t]{4}{*}{ SPEC } & 50 & 20 & 921 & -0.0222 & 0.0024 & -0.0262 \\
\hline & & & & 40 & 929 & -0.0315 & 0.0040 & -0.0389 \\
\hline & & & 100 & 20 & 921 & -0.0230 & 0.0024 & -0.0269 \\
\hline & & & & 40 & 927 & -0.0394 & 0.0028 & -0.0441 \\
\hline .4 & 400 & SPEC \& GEN & 50 & 20 & 704 & -0.0642 & 0.0070 & -0.0757 \\
\hline & & & & 40 & 675 & -0.1088 & 0.0095 & -0.1229 \\
\hline & & & 100 & 20 & 59 & -0.0627 & 0.0068 & -0.0742 \\
\hline & & & & 40 & 691 & -0.1131 & 0.0079 & -0.1265 \\
\hline & & SPEC & 50 & 20 & 904 & -0.0446 & 0.0065 & -0.0546 \\
\hline & & & & 40 & 759 & -0.0737 & 0.0074 & -0.0858 \\
\hline & & & 100 & 20 & 899 & -0.0458 & 0.0061 & -0.0555 \\
\hline & & & & 40 & 903 & -0.0860 & 0.0074 & -0.0979 \\
\hline & 800 & SPEC \& GEN & 50 & 20 & 763 & -0.0631 & 0.0050 & -0.0713 \\
\hline & & & & 40 & 743 & -0.1085 & 0.0074 & -0.1195 \\
\hline & & & 100 & 20 & 31 & -0.0633 & 0.0044 & -0.0713 \\
\hline & & & & 40 & 748 & -0.1122 & 0.0051 & -0.1204 \\
\hline & & SPEC & 50 & 20 & 919 & -0.0438 & 0.0039 & -0.0504 \\
\hline & & & & 40 & 899 & -0.0733 & 0.0048 & -0.0813 \\
\hline & & & 100 & 20 & 915 & -0.0453 & 0.0036 & -0.0513 \\
\hline & & & & 40 & 919 & -0.0850 & 0.0048 & -0.0930 \\
\hline & 1200 & SPEC \& GEN & 50 & 20 & 781 & -0.0629 & 0.0043 & -0.0698 \\
\hline & & & & 40 & 779 & -0.1086 & 0.0067 & -0.1180 \\
\hline & & & 100 & 20 & 22 & -0.0621 & 0.0032 & -0.0661 \\
\hline & & & & 40 & 775 & -0.1120 & 0.0042 & -0.1189 \\
\hline & & SPEC & 50 & 20 & 924 & -0.0438 & 0.0032 & -0.0492 \\
\hline & & & & 40 & 923 & -0.0735 & 0.0039 & -0.0798 \\
\hline & & & 100 & 20 & 922 & -0.0454 & 0.0030 & -0.0505 \\
\hline & & & & 40 & 924 & -0.0853 & 0.0037 & -0.0913 \\
\hline
\end{tabular}


Table 4

Means, Standard Deviations, and $5^{\text {th }}$ Percentiles for $\Delta M c$ Donald's (Complex Model) by Condition

\begin{tabular}{|c|c|c|c|c|c|c|c|c|}
\hline Loading & $n_{\text {Model }}$ & Location & Specific \% & Total items \% & $n$ & $M$ & $S D$ & $5^{\text {th }}$ Percentile \\
\hline \multirow[t]{24}{*}{.2} & \multirow[t]{8}{*}{400} & \multirow[t]{4}{*}{ SPEC \& GEN } & \multirow[t]{2}{*}{50} & 20 & 1000 & -0.2248 & 0.0162 & -0.2519 \\
\hline & & & & 40 & 676 & -0.2273 & 0.0240 & -0.2668 \\
\hline & & & \multirow[t]{2}{*}{100} & 20 & 1000 & -0.2276 & 0.0163 & -0.2546 \\
\hline & & & & 40 & 1000 & -0.3236 & 0.0185 & -0.3531 \\
\hline & & \multirow[t]{4}{*}{ SPEC } & \multirow[t]{2}{*}{50} & 20 & 1000 & -0.1927 & 0.0177 & -0.2224 \\
\hline & & & & 40 & 855 & -0.1697 & 0.0251 & -0.2118 \\
\hline & & & \multirow[t]{2}{*}{100} & 20 & 1000 & -0.1967 & 0.0178 & -0.2255 \\
\hline & & & & 40 & 1000 & -0.2682 & 0.0203 & -0.3018 \\
\hline & \multirow[t]{8}{*}{800} & \multirow[t]{4}{*}{ SPEC \& GEN } & \multirow[t]{2}{*}{50} & 20 & 1000 & -0.2263 & 0.0114 & -0.2460 \\
\hline & & & & 40 & 816 & -0.2298 & 0.0134 & -0.2516 \\
\hline & & & \multirow[t]{2}{*}{100} & 20 & 1000 & -0.2295 & 0.0106 & -0.2473 \\
\hline & & & & 40 & 1000 & -0.3250 & 0.0128 & -0.3456 \\
\hline & & \multirow[t]{4}{*}{ SPEC } & \multirow[t]{2}{*}{50} & 20 & 1000 & -0.1946 & 0.0122 & -0.2158 \\
\hline & & & & 40 & 962 & -0.1711 & 0.0144 & -0.1958 \\
\hline & & & \multirow[t]{2}{*}{100} & 20 & 1000 & -0.1992 & 0.0117 & -0.2190 \\
\hline & & & & 40 & 1000 & -0.2707 & 0.0138 & -0.2941 \\
\hline & \multirow[t]{8}{*}{1200} & \multirow[t]{4}{*}{ SPEC \& GEN } & \multirow[t]{2}{*}{50} & 20 & 1000 & -0.2272 & 0.0088 & -0.2420 \\
\hline & & & & 40 & 886 & -0.2306 & 0.0100 & -0.2470 \\
\hline & & & \multirow[t]{2}{*}{100} & 20 & 1000 & -0.2297 & 0.0089 & -0.2443 \\
\hline & & & & 40 & 1000 & -0.3255 & 0.0098 & -0.3415 \\
\hline & & \multirow[t]{4}{*}{ SPEC } & 50 & 20 & 1000 & -0.1950 & 0.0094 & -0.2112 \\
\hline & & & & 40 & 992 & -0.1714 & 0.0108 & -0.1901 \\
\hline & & & 100 & 20 & 1000 & -0.1994 & 0.0095 & -0.2148 \\
\hline & & & & 40 & 1000 & -0.2706 & 0.0110 & -0.2879 \\
\hline .4 & 400 & SPEC \& GEN & 50 & 20 & 1000 & -0.3512 & 0.0185 & -0.3831 \\
\hline & & & & 40 & 348 & -0.3128 & 0.0242 & -0.3559 \\
\hline & & & 100 & 20 & 1000 & -0.3540 & 0.0178 & -0.3821 \\
\hline & & & & 40 & 1000 & -0.5262 & 0.0195 & -0.5574 \\
\hline & & SPEC & 50 & 20 & 1000 & -0.2960 & 0.0211 & -0.3304 \\
\hline & & & & 40 & 342 & -0.1811 & 0.0258 & -0.2225 \\
\hline & & & 100 & 20 & 1000 & -0.3004 & 0.0203 & -0.3338 \\
\hline & & & & 40 & 1000 & -0.4495 & 0.0222 & -0.4859 \\
\hline & 800 & SPEC \& GEN & 50 & 20 & 1000 & -0.3529 & 0.0129 & -0.3748 \\
\hline & & & & 40 & 374 & -0.3145 & 0.0136 & -0.3359 \\
\hline & & & 100 & 20 & 1000 & -0.3562 & 0.0117 & -0.3754 \\
\hline & & & & 40 & 1000 & -0.5280 & 0.0137 & -0.5495 \\
\hline & & SPEC & 50 & 20 & 1000 & -0.2983 & 0.0146 & -0.3228 \\
\hline & & & & 40 & 423 & -0.1811 & 0.0154 & -0.2050 \\
\hline & & & 100 & 20 & 1000 & -0.3036 & 0.0134 & -0.3260 \\
\hline & & & & 40 & 1000 & -0.4528 & 0.0152 & -0.4784 \\
\hline & 1200 & SPEC \& GEN & 50 & 20 & 1000 & -0.3543 & 0.0101 & -0.3714 \\
\hline & & & & 40 & 422 & -0.3159 & 0.0111 & -0.3339 \\
\hline & & & 100 & 20 & 1000 & -0.3566 & 0.0100 & -0.3724 \\
\hline & & & & 40 & 1000 & -0.5289 & 0.0105 & -0.5457 \\
\hline & & SPEC & 50 & 20 & 1000 & -0.2992 & 0.0113 & -0.3182 \\
\hline & & & & 40 & 462 & -0.1814 & 0.0113 & -0.2003 \\
\hline & & & 100 & 20 & 1000 & -0.3040 & 0.0110 & -0.3222 \\
\hline & & & & 40 & 1000 & -0.4530 & 0.0121 & -0.4720 \\
\hline
\end{tabular}


Table 5

Means, Standard Deviations, and $5^{\text {th }}$ Percentiles for $\Delta M c D o n a l d ' s$ (Simple Model) by Condition

\begin{tabular}{|c|c|c|c|c|c|c|c|c|}
\hline Loading & $n_{\text {Model }}$ & Location & Specific \% & Total items \% & $n$ & $M$ & $S D$ & $5^{\text {th }}$ Percentile \\
\hline \multirow[t]{24}{*}{.2} & \multirow[t]{8}{*}{400} & \multirow[t]{4}{*}{ SPEC \& GEN } & \multirow[t]{2}{*}{50} & 20 & 707 & -0.1531 & 0.0285 & -0.2017 \\
\hline & & & & 40 & 694 & -0.2358 & 0.0359 & -0.2932 \\
\hline & & & \multirow[t]{2}{*}{100} & 20 & 554 & -0.1516 & 0.0251 & -0.1957 \\
\hline & & & & 40 & 686 & -0.2503 & 0.0281 & -0.2957 \\
\hline & & \multirow[t]{4}{*}{ SPEC } & \multirow[t]{2}{*}{50} & 20 & 845 & -0.1129 & 0.0263 & -0.1553 \\
\hline & & & & 40 & 888 & -0.1545 & 0.0303 & -0.2036 \\
\hline & & & \multirow[t]{2}{*}{100} & 20 & 844 & -0.1154 & 0.0261 & -0.1601 \\
\hline & & & & 40 & 899 & -0.1887 & 0.0281 & -0.2360 \\
\hline & \multirow[t]{8}{*}{800} & \multirow[t]{4}{*}{ SPEC \& GEN } & \multirow[t]{2}{*}{50} & 20 & 766 & -0.1483 & 0.0193 & -0.1783 \\
\hline & & & & 40 & 749 & -0.2319 & 0.0271 & -0.2836 \\
\hline & & & \multirow[t]{2}{*}{100} & 20 & 636 & -0.1466 & 0.0163 & -0.1709 \\
\hline & & & & 40 & 746 & -0.2453 & 0.0170 & -0.2721 \\
\hline & & \multirow[t]{4}{*}{ SPEC } & \multirow[t]{2}{*}{50} & 20 & 903 & -0.1081 & 0.0146 & -0.1321 \\
\hline & & & & 40 & 917 & -0.1505 & 0.0210 & -0.1884 \\
\hline & & & \multirow[t]{2}{*}{100} & 20 & 897 & -0.1118 & 0.0143 & -0.1349 \\
\hline & & & & 40 & 915 & -0.1851 & 0.0162 & -0.2123 \\
\hline & \multirow[t]{8}{*}{1200} & \multirow[t]{4}{*}{ SPEC \& GEN } & \multirow[t]{2}{*}{50} & 20 & 780 & -0.1467 & 0.0156 & -0.1713 \\
\hline & & & & 40 & 777 & -0.2306 & 0.0216 & -0.2727 \\
\hline & & & \multirow[t]{2}{*}{100} & 20 & 694 & -0.1456 & 0.0120 & -0.1647 \\
\hline & & & & 40 & 782 & -0.2445 & 0.0140 & -0.2665 \\
\hline & & \multirow[t]{4}{*}{ SPEC } & 50 & 20 & 921 & -0.1072 & 0.0114 & -0.1260 \\
\hline & & & & 40 & 929 & -0.1498 & 0.0182 & -0.1830 \\
\hline & & & 100 & 20 & 921 & -0.1112 & 0.0113 & -0.1293 \\
\hline & & & & 40 & 927 & -0.1855 & 0.0126 & -0.2064 \\
\hline .4 & 400 & SPEC \& GEN & 50 & 20 & 704 & $\begin{array}{l}-0.2908 \\
\end{array}$ & 0.0295 & -0.3389 \\
\hline & & & & 40 & 675 & -0.4578 & 0.0345 & -0.5106 \\
\hline & & & 100 & 20 & 59 & -0.2845 & 0.0290 & -0.3388 \\
\hline & & & & 40 & 691 & -0.4726 & 0.0289 & -0.5203 \\
\hline & & SPEC & 50 & 20 & 904 & -0.2080 & 0.0289 & -0.2523 \\
\hline & & & & 40 & 759 & -0.3278 & 0.0305 & -0.3765 \\
\hline & & & 100 & 20 & 899 & -0.2132 & 0.0274 & -0.2576 \\
\hline & & & & 40 & 903 & -0.3746 & 0.0294 & -0.4213 \\
\hline & 800 & SPEC \& GEN & 50 & 20 & 763 & -0.2862 & 0.0209 & -0.3202 \\
\hline & & & & 40 & 743 & -0.4564 & 0.0261 & -0.4952 \\
\hline & & & 100 & 20 & 31 & -0.2874 & 0.0187 & -0.3234 \\
\hline & & & & 40 & 748 & -0.4691 & 0.0181 & -0.4983 \\
\hline & & SPEC & 50 & 20 & 919 & -0.2045 & 0.0175 & -0.2341 \\
\hline & & & & 40 & 899 & -0.3264 & 0.0195 & -0.3585 \\
\hline & & & 100 & 20 & 915 & -0.2113 & 0.0158 & -0.2372 \\
\hline & & & & 40 & 919 & -0.3714 & 0.0185 & -0.4024 \\
\hline & 1200 & SPEC \& GEN & 50 & 20 & 781 & -0.2854 & 0.0177 & -0.3140 \\
\hline & & & & 40 & 779 & -0.4566 & 0.0234 & -0.4889 \\
\hline & & & 100 & 20 & 22 & -0.2825 & 0.0139 & -0.2989 \\
\hline & & & & 40 & 775 & -0.4684 & 0.0147 & -0.4919 \\
\hline & & SPEC & 50 & 20 & 924 & -0.2046 & 0.0142 & -0.2287 \\
\hline & & & & 40 & 923 & -0.3274 & 0.0158 & -0.3524 \\
\hline & & & 100 & 20 & 922 & -0.2116 & 0.0131 & -0.2340 \\
\hline & & & & 40 & 924 & -0.3725 & 0.0142 & -0.3952 \\
\hline
\end{tabular}


Table 6

\begin{tabular}{|c|c|c|c|c|c|c|c|c|}
\hline Loading & $n_{\text {Model }}$ & Location & Specific $\%$ & Total items $\%$ & $n$ & $M$ & $S D$ & $5^{\text {th }}$ Percentile \\
\hline \multirow[t]{24}{*}{.2} & \multirow[t]{8}{*}{400} & \multirow[t]{4}{*}{ SPEC \& GEN } & \multirow[t]{2}{*}{50} & 20 & 1000 & -0.0098 & 0.0009 & -0.0112 \\
\hline & & & & 40 & 676 & -0.0111 & 0.0011 & -0.0130 \\
\hline & & & \multirow[t]{2}{*}{100} & 20 & 1000 & -0.0100 & 0.0009 & -0.0115 \\
\hline & & & & 40 & 1000 & -0.0172 & 0.0012 & -0.0191 \\
\hline & & \multirow[t]{4}{*}{ SPEC } & \multirow[t]{2}{*}{50} & 20 & 1000 & -0.0081 & 0.0009 & -0.0096 \\
\hline & & & & 40 & 855 & -0.0076 & 0.0011 & -0.0093 \\
\hline & & & \multirow[t]{2}{*}{100} & 20 & 1000 & -0.0082 & 0.0009 & -0.0096 \\
\hline & & & & 40 & 1000 & -0.0130 & 0.0012 & -0.0151 \\
\hline & \multirow[t]{8}{*}{800} & \multirow[t]{4}{*}{ SPEC \& GEN } & \multirow[t]{2}{*}{50} & 20 & 1000 & -0.0099 & 0.0006 & -0.0110 \\
\hline & & & & 40 & 816 & -0.0113 & 0.0007 & -0.0124 \\
\hline & & & \multirow[t]{2}{*}{100} & 20 & 1000 & -0.0101 & 0.0006 & -0.0110 \\
\hline & & & & 40 & 1000 & -0.0172 & 0.0009 & -0.0187 \\
\hline & & \multirow[t]{4}{*}{ SPEC } & \multirow[t]{2}{*}{50} & 20 & 1000 & -0.0081 & 0.0006 & -0.0092 \\
\hline & & & & 40 & 962 & -0.0077 & 0.0007 & -0.0088 \\
\hline & & & \multirow[t]{2}{*}{100} & 20 & 1000 & -0.0084 & 0.0006 & -0.0093 \\
\hline & & & & 40 & 1000 & -0.0131 & 0.0008 & -0.0145 \\
\hline & \multirow[t]{8}{*}{1200} & \multirow[t]{4}{*}{ SPEC \& GEN } & \multirow[t]{2}{*}{50} & 20 & 1000 & -0.0100 & 0.0005 & -0.0107 \\
\hline & & & & 40 & 886 & -0.0113 & 0.0005 & -0.0122 \\
\hline & & & \multirow[t]{2}{*}{100} & 20 & 1000 & -0.0101 & 0.0005 & -0.0109 \\
\hline & & & & 40 & 1000 & -0.0173 & 0.0006 & -0.0183 \\
\hline & & \multirow[t]{4}{*}{ SPEC } & 50 & 20 & 1000 & -0.0082 & 0.0005 & -0.0090 \\
\hline & & & & 40 & 992 & -0.0077 & 0.0005 & -0.0085 \\
\hline & & & 100 & 20 & 1000 & -0.0084 & 0.0005 & -0.0092 \\
\hline & & & & 40 & 1000 & -0.0131 & 0.0007 & -0.0142 \\
\hline .4 & 400 & SPEC \& GEN & 50 & 20 & 1000 & $\begin{array}{l}-0.0171 \\
\end{array}$ & 0.0011 & $\begin{array}{l}-0.0191 \\
\end{array}$ \\
\hline & & & & 40 & 348 & -0.0169 & 0.0013 & -0.0192 \\
\hline & & & 100 & 20 & 1000 & -0.0173 & 0.0011 & -0.0192 \\
\hline & & & & 40 & 1000 & -0.0345 & 0.0018 & -0.0374 \\
\hline & & SPEC & 50 & 20 & 1000 & -0.0136 & 0.0012 & -0.0156 \\
\hline & & & & 40 & 342 & -0.0085 & 0.0012 & -0.0104 \\
\hline & & & 100 & 20 & 1000 & -0.0138 & 0.0012 & -0.0156 \\
\hline & & & & 40 & 1000 & -0.0261 & 0.0018 & -0.0291 \\
\hline & 800 & SPEC \& GEN & 50 & 20 & 1000 & -0.0172 & 0.0008 & -0.0186 \\
\hline & & & & 40 & 374 & -0.0171 & 0.0008 & -0.0183 \\
\hline & & & 100 & 20 & 1000 & -0.0174 & 0.0007 & -0.0187 \\
\hline & & & & 40 & 1000 & -0.0345 & 0.0013 & -0.0365 \\
\hline & & SPEC & 50 & 20 & 1000 & -0.0137 & 0.0008 & -0.0151 \\
\hline & & & & 40 & 423 & -0.0085 & 0.0007 & -0.0097 \\
\hline & & & 100 & 20 & 1000 & -0.0140 & 0.0008 & -0.0153 \\
\hline & & & & 40 & 1000 & -0.0262 & 0.0012 & -0.0284 \\
\hline & 1200 & SPEC \& GEN & 50 & 20 & 1000 & -0.0173 & 0.0006 & -0.0183 \\
\hline & & & & 40 & 422 & -0.0172 & 0.0006 & -0.0182 \\
\hline & & & 100 & 20 & 1000 & -0.0175 & 0.0006 & -0.0185 \\
\hline & & & & 40 & 1000 & -0.0346 & 0.0010 & -0.0361 \\
\hline & & SPEC & 50 & 20 & 1000 & -0.0137 & 0.0006 & -0.0148 \\
\hline & & & & 40 & 462 & -0.0086 & 0.0005 & -0.0095 \\
\hline & & & 100 & 20 & 1000 & -0.0140 & 0.0006 & -0.0151 \\
\hline & & & & 40 & 1000 & -0.0262 & 0.0010 & -0.0279 \\
\hline
\end{tabular}


Table 7

Means, Standard Deviations, and $5^{\text {th }}$ Percentiles for $\triangle C F I$ (Simple Model) by Condition

\begin{tabular}{|c|c|c|c|c|c|c|c|c|}
\hline Loading & $n_{\text {Model }}$ & Location & Specific \% & Total items $\%$ & $n$ & $M$ & $S D$ & $5^{\text {th }}$ Percentile \\
\hline \multirow[t]{24}{*}{.2} & \multirow{8}{*}{400} & \multirow{4}{*}{ SPEC \& GEN } & \multirow[t]{2}{*}{50} & 20 & 707 & -0.0052 & 0.0010 & -0.0069 \\
\hline & & & & 40 & 694 & -0.0096 & 0.0016 & -0.0124 \\
\hline & & & \multirow[t]{2}{*}{100} & 20 & 554 & -0.0052 & 0.0009 & -0.0067 \\
\hline & & & & 40 & 686 & -0.0104 & 0.0012 & -0.0125 \\
\hline & & \multirow[t]{4}{*}{ SPEC } & \multirow[t]{2}{*}{50} & 20 & 845 & -0.0037 & 0.0008 & -0.0051 \\
\hline & & & & 40 & 888 & -0.0057 & 0.0012 & -0.0076 \\
\hline & & & \multirow[t]{2}{*}{100} & 20 & 844 & -0.0038 & 0.0008 & -0.0052 \\
\hline & & & & 40 & 899 & -0.0072 & 0.0011 & -0.0090 \\
\hline & \multirow[t]{8}{*}{800} & \multirow{4}{*}{ SPEC \& GEN } & \multirow[t]{2}{*}{50} & 20 & 766 & -0.0052 & 0.0007 & -0.0063 \\
\hline & & & & 40 & 749 & -0.0096 & 0.0013 & -0.0121 \\
\hline & & & \multirow[t]{2}{*}{100} & 20 & 636 & -0.0051 & 0.0006 & -0.0060 \\
\hline & & & & 40 & 746 & -0.0103 & 0.0007 & -0.0115 \\
\hline & & \multirow[t]{4}{*}{ SPEC } & \multirow[t]{2}{*}{50} & 20 & 903 & -0.0036 & 0.0005 & -0.0044 \\
\hline & & & & 40 & 917 & -0.0057 & 0.0008 & -0.0072 \\
\hline & & & \multirow[t]{2}{*}{100} & 20 & 897 & -0.0038 & 0.0005 & -0.0046 \\
\hline & & & & 40 & 915 & -0.0072 & 0.0006 & -0.0082 \\
\hline & \multirow[t]{8}{*}{1200} & \multirow[t]{4}{*}{ SPEC \& GEN } & \multirow[t]{2}{*}{50} & 20 & 780 & -0.0052 & 0.0006 & -0.0061 \\
\hline & & & & 40 & 777 & -0.0096 & 0.0010 & -0.0116 \\
\hline & & & \multirow[t]{2}{*}{100} & 20 & 694 & -0.0051 & 0.0004 & -0.0058 \\
\hline & & & & 40 & 782 & -0.0103 & 0.0006 & -0.0113 \\
\hline & & \multirow[t]{4}{*}{ SPEC } & 50 & 20 & 921 & -0.0036 & 0.0004 & -0.0042 \\
\hline & & & & 40 & 929 & -0.0057 & 0.0007 & -0.0071 \\
\hline & & & 100 & 20 & 921 & -0.0038 & 0.0004 & -0.0044 \\
\hline & & & & 40 & 927 & -0.0072 & 0.0005 & -0.0081 \\
\hline .4 & 400 & SPEC \& GEN & 50 & 20 & 704 & -0.0113 & 0.0012 & -0.0134 \\
\hline & & & & 40 & 675 & -0.0231 & 0.0021 & -0.0263 \\
\hline & & & 100 & 20 & 59 & -0.0111 & 0.0012 & -0.0135 \\
\hline & & & & 40 & 691 & -0.0243 & 0.0018 & -0.0272 \\
\hline & & SPEC & 50 & 20 & 904 & -0.0076 & 0.0011 & -0.0092 \\
\hline & & & & 40 & 759 & -0.0144 & 0.0015 & -0.0169 \\
\hline & & & 100 & 20 & 899 & -0.0078 & 0.0010 & -0.0094 \\
\hline & & & & 40 & 903 & -0.0172 & 0.0015 & -0.0197 \\
\hline & 800 & SPEC \& GEN & 50 & 20 & 763 & -0.0113 & 0.0009 & -0.0128 \\
\hline & & & & 40 & 743 & -0.0232 & 0.0017 & -0.0257 \\
\hline & & & 100 & 20 & 31 & -0.0112 & 0.0007 & -0.0124 \\
\hline & & & & 40 & 748 & -0.0242 & 0.0012 & -0.0261 \\
\hline & & SPEC & 50 & 20 & 919 & -0.0075 & 0.0007 & -0.0087 \\
\hline & & & & 40 & 899 & -0.0144 & 0.0010 & -0.0161 \\
\hline & & & 100 & 20 & 915 & -0.0078 & 0.0006 & -0.0088 \\
\hline & & & & 40 & 919 & -0.0171 & 0.0010 & -0.0188 \\
\hline & 1200 & SPEC \& GEN & 50 & 20 & 781 & -0.0113 & 0.0008 & -0.0126 \\
\hline & & & & 40 & 779 & -0.0233 & 0.0016 & -0.0255 \\
\hline & & & 100 & 20 & 22 & -0.0111 & 0.0006 & -0.0119 \\
\hline & & & & 40 & 775 & -0.0242 & 0.0009 & -0.0258 \\
\hline & & SPEC & 50 & 20 & 924 & -0.0075 & 0.0006 & -0.0085 \\
\hline & & & & 40 & 923 & -0.0145 & 0.0008 & -0.0158 \\
\hline & & & 100 & 20 & 922 & -0.0078 & 0.0005 & -0.0087 \\
\hline & & & & 40 & 924 & -0.0171 & 0.0008 & -0.0184 \\
\hline
\end{tabular}


Table 8

Means, Standard Deviations, and $95^{\text {th }}$ Percentiles for $\triangle S R M R$ (Complex Model) by Condition

\begin{tabular}{|c|c|c|c|c|c|c|c|c|}
\hline Loading & $n_{\text {Model }}$ & Location & Specific $\%$ & Total items $\%$ & $n$ & $M$ & $S D$ & $95^{\text {th }}$ Percentile \\
\hline \multirow[t]{24}{*}{.2} & \multirow[t]{8}{*}{400} & \multirow[t]{4}{*}{ SPEC \& GEN } & \multirow[t]{2}{*}{50} & 20 & 1000 & 0.9348 & 0.0686 & 1.0502 \\
\hline & & & & 40 & 676 & 0.8895 & 0.0623 & 0.9931 \\
\hline & & & \multirow[t]{2}{*}{100} & 20 & 1000 & 0.9375 & 0.0688 & 1.0536 \\
\hline & & & & 40 & 1000 & 0.9729 & 0.0643 & 1.0798 \\
\hline & & \multirow[t]{4}{*}{ SPEC } & \multirow[t]{2}{*}{50} & 20 & 1000 & 0.8913 & 0.0729 & 1.0129 \\
\hline & & & & 40 & 855 & 0.8217 & 0.0693 & 0.9378 \\
\hline & & & \multirow[t]{2}{*}{100} & 20 & 1000 & 0.8944 & 0.0731 & 1.0186 \\
\hline & & & & 40 & 1000 & 0.8897 & 0.0722 & 1.0119 \\
\hline & \multirow[t]{8}{*}{800} & \multirow[t]{4}{*}{ SPEC \& GEN } & \multirow[t]{2}{*}{50} & 20 & 1000 & 0.9428 & 0.0483 & 1.0226 \\
\hline & & & & 40 & 816 & 0.8947 & 0.0437 & 0.9692 \\
\hline & & & \multirow[t]{2}{*}{100} & 20 & 1000 & 0.9456 & 0.0480 & 1.0264 \\
\hline & & & & 40 & 1000 & 0.9804 & 0.0447 & 1.0546 \\
\hline & & \multirow[t]{4}{*}{ SPEC } & \multirow[t]{2}{*}{50} & 20 & 1000 & 0.8995 & 0.0512 & 0.9858 \\
\hline & & & & 40 & 962 & 0.8299 & 0.0488 & 0.9119 \\
\hline & & & \multirow[t]{2}{*}{100} & 20 & 1000 & 0.9027 & 0.0511 & 0.9887 \\
\hline & & & & 40 & 1000 & 0.8976 & 0.0504 & 0.9808 \\
\hline & \multirow[t]{8}{*}{1200} & \multirow[t]{4}{*}{ SPEC \& GEN } & \multirow[t]{2}{*}{50} & 20 & 1000 & 0.9430 & 0.0381 & 1.0070 \\
\hline & & & & 40 & 886 & 0.8960 & 0.0341 & 0.9542 \\
\hline & & & \multirow[t]{2}{*}{100} & 20 & 1000 & 0.9453 & 0.0380 & 1.0097 \\
\hline & & & & 40 & 1000 & 0.9806 & 0.0352 & 1.0409 \\
\hline & & \multirow[t]{4}{*}{ SPEC } & 50 & 20 & 1000 & 0.8992 & 0.0406 & 0.9672 \\
\hline & & & & 40 & 992 & 0.8291 & 0.0389 & 0.8944 \\
\hline & & & 100 & 20 & 1000 & 0.9021 & 0.0406 & 0.9700 \\
\hline & & & & 40 & 1000 & 0.8970 & 0.0399 & 0.9644 \\
\hline .4 & 400 & SPEC \& GEN & 50 & 20 & 1000 & 0.9885 & 0.0630 & 1.0937 \\
\hline & & & & 40 & 348 & 0.8734 & 0.0539 & 0.9627 \\
\hline & & & 100 & 20 & 1000 & 0.9930 & 0.0632 & 1.0982 \\
\hline & & & & 40 & 1000 & 1.0682 & 0.0552 & 1.1592 \\
\hline & & SPEC & 50 & 20 & 1000 & 0.9008 & 0.0722 & 1.0225 \\
\hline & & & & 40 & 342 & 0.7392 & 0.0653 & 0.8515 \\
\hline & & & 100 & 20 & 1000 & 0.9057 & 0.0725 & 1.0292 \\
\hline & & & & 40 & 1000 & 0.9093 & 0.0712 & 1.0305 \\
\hline & 800 & SPEC \& GEN & 50 & 20 & 1000 & 0.9960 & 0.0446 & 1.0704 \\
\hline & & & & 40 & 374 & 0.8780 & 0.0364 & 0.9378 \\
\hline & & & 100 & 20 & 1000 & 1.0006 & 0.0440 & 1.0749 \\
\hline & & & & 40 & 1000 & 1.0750 & 0.0383 & 1.1405 \\
\hline & & SPEC & 50 & 20 & 1000 & 0.9090 & 0.0510 & 0.9933 \\
\hline & & & & 40 & 423 & 0.7424 & 0.0431 & 0.8115 \\
\hline & & & 100 & 20 & 1000 & 0.9140 & 0.0507 & 0.9993 \\
\hline & & & & 40 & 1000 & 0.9170 & 0.0497 & 0.9978 \\
\hline & 1200 & SPEC \& GEN & 50 & 20 & 1000 & 0.9968 & 0.0351 & 1.0544 \\
\hline & & & & 40 & 422 & 0.8801 & 0.0293 & 0.9300 \\
\hline & & & 100 & 20 & 1000 & 1.0007 & 0.0347 & 1.0600 \\
\hline & & & & 40 & 1000 & 1.0760 & 0.0301 & 1.1260 \\
\hline & & SPEC & 50 & 20 & 1000 & 0.9089 & 0.0404 & 0.9763 \\
\hline & & & & 40 & 462 & 0.7439 & 0.0355 & 0.8046 \\
\hline & & & 100 & 20 & 1000 & 0.9134 & 0.0402 & 0.9818 \\
\hline & & & & 40 & 1000 & 0.9166 & 0.0394 & 0.9833 \\
\hline
\end{tabular}


Table 9

\begin{tabular}{|c|c|c|c|c|c|c|c|c|}
\hline Loading & $n_{\text {Model }}$ & Location & Specific $\%$ & Total items $\%$ & $n$ & $M$ & $S D$ & $95^{\text {th }}$ Percentile \\
\hline \multirow[t]{24}{*}{.2} & \multirow[t]{8}{*}{400} & \multirow[t]{4}{*}{ SPEC \& GEN } & \multirow[t]{2}{*}{50} & 20 & 707 & 0.2615 & 0.0623 & 0.3377 \\
\hline & & & & 40 & 694 & 0.3961 & 0.2174 & 0.8755 \\
\hline & & & \multirow[t]{2}{*}{100} & 20 & 554 & 0.2615 & 0.0583 & 0.3201 \\
\hline & & & & 40 & 686 & 0.2761 & 0.0649 & 0.3312 \\
\hline & & \multirow[t]{4}{*}{ SPEC } & \multirow[t]{2}{*}{50} & 20 & 845 & 0.2355 & 0.0389 & 0.2996 \\
\hline & & & & 40 & 888 & 0.3703 & 0.2146 & 0.7716 \\
\hline & & & \multirow[t]{2}{*}{100} & 20 & 844 & 0.2386 & 0.0441 & 0.2988 \\
\hline & & & & 40 & 899 & 0.2466 & 0.0445 & 0.3086 \\
\hline & \multirow[t]{8}{*}{800} & \multirow[t]{4}{*}{ SPEC \& GEN } & \multirow[t]{2}{*}{50} & 20 & 766 & 0.2571 & 0.0488 & 0.3180 \\
\hline & & & & 40 & 749 & 0.3781 & 0.2004 & 0.8509 \\
\hline & & & \multirow[t]{2}{*}{100} & 20 & 636 & 0.2565 & 0.0519 & 0.2975 \\
\hline & & & & 40 & 746 & 0.2675 & 0.0312 & 0.3052 \\
\hline & & \multirow[t]{4}{*}{ SPEC } & \multirow[t]{2}{*}{50} & 20 & 903 & 0.2294 & 0.0343 & 0.2778 \\
\hline & & & & 40 & 917 & 0.3523 & 0.2136 & 0.7502 \\
\hline & & & \multirow[t]{2}{*}{100} & 20 & 897 & 0.2331 & 0.0313 & 0.2764 \\
\hline & & & & 40 & 915 & 0.2403 & 0.0300 & 0.2820 \\
\hline & \multirow[t]{8}{*}{1200} & \multirow{4}{*}{ SPEC \& GEN } & \multirow[t]{2}{*}{50} & 20 & 780 & 0.2564 & 0.0476 & 0.3073 \\
\hline & & & & 40 & 777 & 0.3598 & 0.1797 & 0.8452 \\
\hline & & & \multirow[t]{2}{*}{100} & 20 & 694 & 0.2530 & 0.0292 & 0.2857 \\
\hline & & & & 40 & 782 & 0.2709 & 0.0550 & 0.2973 \\
\hline & & \multirow{4}{*}{ SPEC } & 50 & 20 & 921 & 0.2274 & 0.0297 & 0.2663 \\
\hline & & & & 40 & 929 & 0.3323 & 0.2060 & 0.7365 \\
\hline & & & 100 & 20 & 921 & 0.2313 & 0.0198 & 0.2630 \\
\hline & & & & 40 & 927 & 0.2401 & 0.0328 & 0.2703 \\
\hline .4 & 400 & SPEC \& GEN & 50 & 20 & 704 & 0.3278 & 0.0784 & 0.4161 \\
\hline & & & & 40 & 675 & 0.6656 & 0.2783 & 1.0255 \\
\hline & & & 100 & 20 & 59 & 0.3213 & 0.0346 & 0.3991 \\
\hline & & & & 40 & 691 & 0.3652 & 0.0646 & 0.4143 \\
\hline & & SPEC & 50 & 20 & 904 & 0.2277 & 0.0524 & 0.2863 \\
\hline & & & & 40 & 759 & 0.7598 & 0.0817 & 0.8880 \\
\hline & & & 100 & 20 & 899 & 0.2517 & 0.0358 & 0.3145 \\
\hline & & & & 40 & 903 & 0.2971 & 0.1161 & 0.3943 \\
\hline & 800 & SPEC \& GEN & 50 & 20 & 763 & 0.3197 & 0.0573 & 0.3953 \\
\hline & & & & 40 & 743 & 0.6839 & 0.2762 & 1.0097 \\
\hline & & & 100 & 20 & 31 & 0.3350 & 0.0996 & 0.3738 \\
\hline & & & & 40 & 748 & 0.3576 & 0.0314 & 0.3937 \\
\hline & & SPEC & 50 & 20 & 919 & 0.2220 & 0.0236 & 0.2626 \\
\hline & & & & 40 & 899 & 0.7623 & 0.0551 & 0.8529 \\
\hline & & & 100 & 20 & 915 & 0.2497 & 0.0246 & 0.2918 \\
\hline & & & & 40 & 919 & 0.2738 & 0.0539 & 0.3117 \\
\hline & 1200 & SPEC \& GEN & 50 & 20 & 781 & 0.3216 & 0.0576 & 0.3861 \\
\hline & & & & 40 & 779 & 0.6842 & 0.2787 & 0.9982 \\
\hline & & & 100 & 20 & 22 & 0.3051 & 0.0174 & 0.3324 \\
\hline & & & & 40 & 775 & 0.3577 & 0.0299 & 0.3861 \\
\hline & & SPEC & 50 & 20 & 924 & 0.2221 & 0.0195 & 0.2530 \\
\hline & & & & 40 & 923 & 0.7652 & 0.0402 & 0.8344 \\
\hline & & & 100 & 20 & 922 & 0.2496 & 0.0268 & 0.2819 \\
\hline & & & & 40 & 924 & 0.2716 & 0.0398 & 0.3014 \\
\hline
\end{tabular}


Table 10

Means, Standard Deviations, and $95^{\text {th }}$ Percentiles for $\triangle R M S E A$ (Complex Model) by Condition

\begin{tabular}{|c|c|c|c|c|c|c|c|c|}
\hline Loading & $n_{\text {Model }}$ & Location & Specific $\%$ & Total items $\%$ & $n$ & $M$ & $S D$ & $95^{\text {th }}$ Percentile \\
\hline \multirow[t]{24}{*}{.2} & \multirow[t]{8}{*}{400} & \multirow[t]{4}{*}{ SPEC \& GEN } & \multirow[t]{2}{*}{50} & 20 & 1000 & 0.0475 & 0.0063 & 0.0563 \\
\hline & & & & 40 & 676 & 0.0477 & 0.0080 & 0.0588 \\
\hline & & & \multirow[t]{2}{*}{100} & 20 & 1000 & 0.0478 & 0.0063 & 0.0567 \\
\hline & & & & 40 & 1000 & 0.0606 & 0.0065 & 0.0697 \\
\hline & & \multirow[t]{4}{*}{ SPEC } & \multirow[t]{2}{*}{50} & 20 & 1000 & 0.0429 & 0.0063 & 0.0523 \\
\hline & & & & 40 & 855 & 0.0396 & 0.0080 & 0.0509 \\
\hline & & & \multirow[t]{2}{*}{100} & 20 & 1000 & 0.0434 & 0.0063 & 0.0529 \\
\hline & & & & 40 & 1000 & 0.0533 & 0.0065 & 0.0630 \\
\hline & \multirow[t]{8}{*}{800} & \multirow[t]{4}{*}{ SPEC \& GEN } & \multirow[t]{2}{*}{50} & 20 & 1000 & 0.0505 & 0.0045 & 0.0566 \\
\hline & & & & 40 & 816 & 0.0509 & 0.0052 & 0.0575 \\
\hline & & & \multirow[t]{2}{*}{100} & 20 & 1000 & 0.0509 & 0.0045 & 0.0567 \\
\hline & & & & 40 & 1000 & 0.0636 & 0.0046 & 0.0696 \\
\hline & & \multirow[t]{4}{*}{ SPEC } & \multirow[t]{2}{*}{50} & 20 & 1000 & 0.0461 & 0.0045 & 0.0523 \\
\hline & & & & 40 & 962 & 0.0425 & 0.0052 & 0.0495 \\
\hline & & & \multirow[t]{2}{*}{100} & 20 & 1000 & 0.0466 & 0.0045 & 0.0527 \\
\hline & & & & 40 & 1000 & 0.0565 & 0.0046 & 0.0627 \\
\hline & \multirow[t]{8}{*}{1200} & \multirow[t]{4}{*}{ SPEC \& GEN } & \multirow[t]{2}{*}{50} & 20 & 1000 & 0.0515 & 0.0038 & 0.0564 \\
\hline & & & & 40 & 886 & 0.0520 & 0.0042 & 0.0571 \\
\hline & & & \multirow[t]{2}{*}{100} & 20 & 1000 & 0.0518 & 0.0038 & 0.0566 \\
\hline & & & & 40 & 1000 & 0.0645 & 0.0040 & 0.0696 \\
\hline & & \multirow[t]{4}{*}{ SPEC } & 50 & 20 & 1000 & 0.0469 & 0.0039 & 0.0520 \\
\hline & & & & 40 & 992 & 0.0435 & 0.0043 & 0.0489 \\
\hline & & & 100 & 20 & 1000 & 0.0476 & 0.0039 & 0.0525 \\
\hline & & & & 40 & 1000 & 0.0573 & 0.0040 & 0.0624 \\
\hline .4 & 400 & SPEC \& GEN & 50 & 20 & 1000 & 0.0642 & 0.0066 & 0.0733 \\
\hline & & & & 40 & 348 & 0.0591 & 0.0080 & 0.0701 \\
\hline & & & 100 & 20 & 1000 & 0.0644 & 0.0065 & 0.0733 \\
\hline & & & & 40 & 1000 & 0.0866 & 0.0068 & 0.0960 \\
\hline & & SPEC & 50 & 20 & 1000 & 0.0570 & 0.0066 & 0.0668 \\
\hline & & & & 40 & 342 & 0.0417 & 0.0080 & 0.0524 \\
\hline & & & 100 & 20 & 1000 & 0.0575 & 0.0066 & 0.0673 \\
\hline & & & & 40 & 1000 & 0.0766 & 0.0069 & 0.0869 \\
\hline & 800 & SPEC \& GEN & 50 & 20 & 1000 & 0.0672 & 0.0047 & 0.0732 \\
\hline & & & & 40 & 374 & 0.0621 & 0.0052 & 0.0685 \\
\hline & & & 100 & 20 & 1000 & 0.0675 & 0.0047 & 0.0734 \\
\hline & & & & 40 & 1000 & 0.0896 & 0.0049 & 0.0960 \\
\hline & & SPEC & 50 & 20 & 1000 & 0.0602 & 0.0047 & 0.0667 \\
\hline & & & & 40 & 423 & 0.0442 & 0.0055 & 0.0513 \\
\hline & & & 100 & 20 & 1000 & 0.0608 & 0.0047 & 0.0670 \\
\hline & & & & 40 & 1000 & 0.0798 & 0.0049 & 0.0864 \\
\hline & 1200 & SPEC \& GEN & 50 & 20 & 1000 & 0.0681 & 0.0040 & 0.0731 \\
\hline & & & & 40 & 422 & 0.0634 & 0.0044 & 0.0685 \\
\hline & & & 100 & 20 & 1000 & 0.0685 & 0.0039 & 0.0734 \\
\hline & & & & 40 & 1000 & 0.0906 & 0.0041 & 0.0959 \\
\hline & & SPEC & 50 & 20 & 1000 & 0.0610 & 0.0040 & 0.0664 \\
\hline & & & & 40 & 462 & 0.0448 & 0.0045 & 0.0503 \\
\hline & & & 100 & 20 & 1000 & 0.0617 & 0.0040 & 0.0668 \\
\hline & & & & 40 & 1000 & 0.0806 & 0.0041 & 0.0860 \\
\hline
\end{tabular}


Table 11

\begin{tabular}{|c|c|c|c|c|c|c|c|c|}
\hline Loading & $n_{\text {Model }}$ & Location & Specific $\%$ & Total items $\%$ & $n$ & $M$ & $S D$ & $95^{\text {th }}$ Percentile \\
\hline \multirow[t]{24}{*}{.2} & \multirow[t]{8}{*}{400} & \multirow[t]{4}{*}{ SPEC \& GEN } & \multirow[t]{2}{*}{50} & 20 & 707 & 0.0395 & 0.0070 & 0.0490 \\
\hline & & & & 40 & 694 & 0.0519 & 0.0073 & 0.0619 \\
\hline & & & \multirow[t]{2}{*}{100} & 20 & 554 & 0.0393 & 0.0066 & 0.0480 \\
\hline & & & & 40 & 686 & 0.0539 & 0.0067 & 0.0624 \\
\hline & & \multirow[t]{4}{*}{ SPEC } & \multirow[t]{2}{*}{50} & 20 & 845 & 0.0322 & 0.0076 & 0.0422 \\
\hline & & & & 40 & 888 & 0.0389 & 0.0078 & 0.0499 \\
\hline & & & \multirow[t]{2}{*}{100} & 20 & 844 & 0.0324 & 0.0077 & 0.0426 \\
\hline & & & & 40 & 899 & 0.0441 & 0.0077 & 0.0540 \\
\hline & \multirow[t]{8}{*}{800} & \multirow[t]{4}{*}{ SPEC \& GEN } & \multirow[t]{2}{*}{50} & 20 & 766 & 0.0405 & 0.0051 & 0.0470 \\
\hline & & & & 40 & 749 & 0.0528 & 0.0053 & 0.0608 \\
\hline & & & \multirow[t]{2}{*}{100} & 20 & 636 & 0.0401 & 0.0047 & 0.0457 \\
\hline & & & & 40 & 746 & 0.0547 & 0.0045 & 0.0602 \\
\hline & & \multirow[t]{4}{*}{ SPEC } & \multirow[t]{2}{*}{50} & 20 & 903 & 0.0331 & 0.0051 & 0.0398 \\
\hline & & & & 40 & 917 & 0.0400 & 0.0055 & 0.0484 \\
\hline & & & \multirow[t]{2}{*}{100} & 20 & 897 & 0.0337 & 0.0051 & 0.0401 \\
\hline & & & & 40 & 915 & 0.0454 & 0.0051 & 0.0518 \\
\hline & \multirow[t]{8}{*}{1200} & \multirow[t]{4}{*}{ SPEC \& GEN } & \multirow[t]{2}{*}{50} & 20 & 780 & 0.0408 & 0.0040 & 0.0461 \\
\hline & & & & 40 & 777 & 0.0532 & 0.0043 & 0.0599 \\
\hline & & & \multirow[t]{2}{*}{100} & 20 & 694 & 0.0408 & 0.0037 & 0.0452 \\
\hline & & & & 40 & 782 & 0.0552 & 0.0038 & 0.0596 \\
\hline & & \multirow[t]{4}{*}{ SPEC } & 50 & 20 & 921 & 0.0336 & 0.0042 & 0.0386 \\
\hline & & & & 40 & 929 & 0.0408 & 0.0047 & 0.0480 \\
\hline & & & 100 & 20 & 921 & 0.0343 & 0.0042 & 0.0394 \\
\hline & & & & 40 & 927 & 0.0462 & 0.0041 & 0.0514 \\
\hline .4 & 400 & SPEC \& GEN & 50 & 20 & 704 & 0.0592 & 0.0066 & 0.0677 \\
\hline & & & & 40 & 675 & 0.0808 & 0.0070 & 0.0894 \\
\hline & & & 100 & 20 & 59 & 0.0575 & 0.0069 & 0.0657 \\
\hline & & & & 40 & 691 & 0.0826 & 0.0068 & 0.0907 \\
\hline & & SPEC & 50 & 20 & 904 & 0.0469 & 0.0076 & 0.0565 \\
\hline & & & & 40 & 759 & 0.0630 & 0.0075 & 0.0726 \\
\hline & & & 100 & 20 & 899 & 0.0474 & 0.0076 & 0.0572 \\
\hline & & & & 40 & 903 & 0.0688 & 0.0075 & 0.0783 \\
\hline & 800 & SPEC \& GEN & 50 & 20 & 763 & 0.0602 & 0.0049 & 0.0664 \\
\hline & & & & 40 & 743 & 0.0818 & 0.0052 & 0.0885 \\
\hline & & & 100 & 20 & 31 & 0.0602 & 0.0047 & 0.0650 \\
\hline & & & & 40 & 748 & 0.0836 & 0.0045 & 0.0891 \\
\hline & & SPEC & 50 & 20 & 919 & 0.0482 & 0.0053 & 0.0552 \\
\hline & & & & 40 & 899 & 0.0644 & 0.0053 & 0.0712 \\
\hline & & & 100 & 20 & 915 & 0.0492 & 0.0050 & 0.0555 \\
\hline & & & & 40 & 919 & 0.0703 & 0.0052 & 0.0767 \\
\hline & 1200 & SPEC \& GEN & 50 & 20 & 781 & 0.0606 & 0.0040 & 0.0658 \\
\hline & & & & 40 & 779 & 0.0824 & 0.0044 & 0.0883 \\
\hline & & & 100 & 20 & 22 & 0.0607 & 0.0043 & 0.0640 \\
\hline & & & & 40 & 775 & 0.0840 & 0.0038 & 0.0886 \\
\hline & & SPEC & 50 & 20 & 924 & 0.0490 & 0.0042 & 0.0544 \\
\hline & & & & 40 & 923 & 0.0655 & 0.0043 & 0.0706 \\
\hline & & & 100 & 20 & 922 & 0.0500 & 0.0042 & 0.0552 \\
\hline & & & & 40 & 924 & 0.0712 & 0.0041 & 0.0762 \\
\hline
\end{tabular}


The simulation cutoff values in Table 12 were determined using the minimum, in terms of absolute value, $95^{\text {th }} / 5^{\text {th }}$ percentiles for each $\Delta \mathrm{GOF}$ index disaggregated by model complexity and sample size. For example, in Table 3 the values used for $\Delta$ Gamma using the complex model with a sample size of 400 was.- .0452 , for a sample size of 800 the value was.- .0418 , and sample size of 1200 the value was -.0404 . These values were chosen because they are the smallest (i.e., minimum) change (in terms of absolute value) to indicate a lack of invariance. Therefore, any values beyond the simulation cutoffs would be flagged as non-invariant. That is, if the difference between the test of configural invariance and metric invariance is beyond the simulated cutoff value then a lack of invariance exists at the factor loading level. Since SRMR and RMSEA are scaled differently, an increase in these two fit indices indicates a lack of metric invariance. 
Table 12

Simulation Cutoff Values by Model Complexity and Sample Size

\begin{tabular}{|c|c|c|c|}
\hline Index & Model & $n_{\text {Model }}$ & Cutoff value \\
\hline \multirow[t]{6}{*}{$\Delta$ Gamma } & Complex & 400 & -.0452 \\
\hline & & 800 & -.0418 \\
\hline & & 1200 & -.0404 \\
\hline & Simple & 400 & -.0322 \\
\hline & & 800 & -.0275 \\
\hline & & 1200 & -.0262 \\
\hline \multirow[t]{6}{*}{$\Delta$ McDonald's } & Complex & 400 & -.2118 \\
\hline & & 800 & -.1958 \\
\hline & & 1200 & -.1901 \\
\hline & Simple & 400 & -.1553 \\
\hline & & 800 & -.1321 \\
\hline & & 1200 & -.1260 \\
\hline \multirow[t]{6}{*}{$\Delta \mathrm{CFI}$} & Complex & 400 & -.0093 \\
\hline & & 800 & -.0088 \\
\hline & & 1200 & -.0085 \\
\hline & Simple & 400 & -.0051 \\
\hline & & 800 & -.0045 \\
\hline & & 1200 & -.0043 \\
\hline \multirow[t]{6}{*}{$\Delta \mathrm{SRMR}$} & Complex & 400 & .8515 \\
\hline & & 800 & .8115 \\
\hline & & 1200 & .8046 \\
\hline & Simple & 400 & .2863 \\
\hline & & 800 & .2626 \\
\hline & & 1200 & .2530 \\
\hline \multirow[t]{6}{*}{$\triangle \mathrm{RMSEA}$} & Complex & 400 & .0509 \\
\hline & & 800 & .0495 \\
\hline & & 1200 & .0489 \\
\hline & Simple & 400 & .0422 \\
\hline & & 800 & .0398 \\
\hline & & 1200 & .0386 \\
\hline
\end{tabular}

Note. Cutoff values used to calculate Power and Type I error 


\section{Type I Error Rates}

After defining the simulation cutoff values, the Type I error rates are calculated. Type I error occurs when the invariant models are being indicated as non-invariant using the previously defined simulation cutoff values from Table 12 . The $\Delta \mathrm{GOF}$ indices were calculated by subtracting the constrained invariant model from the unconstrained invariant model. Any replication that had a larger (absolute value) $\triangle \mathrm{GOF}$ index than the simulated cutoff value was flagged as non-invariant. For example, if using the complex model with sample size of 400, the simulation cutoff value for CFI is -.0093. Any replication without condition codes exceeding the CFI cutoff value would be identified as non-invariant (i.e., Type I error). The Type I error rates for the invariant models were calculated for each index. Presented in the additional supplements files are the six (i.e., simple versus complex models and the three different sample sizes) invariant charts that graph the Type I error for each index. There were only six combinations that could be simulated to be completely invariant, the other conditions (i.e., factor loading differences, total non-invariant items, etc.) are used for the non-invariant models. As shown in the charts all indices have no Type I error except for $\triangle \mathrm{SRMR}$. This occurred because the $\Delta \mathrm{GOF}$ indices in the non-invariant models are much larger than the $\triangle \mathrm{GOF}$ indices in the invariant models (refer to Table 13 to view the summary statistics of the baseline $\Delta \mathrm{GOF}$ indices). Comparing the simulated cutoffs in Table 12 to the invariant models' $\Delta$ GOF indices (Table 13), it is clear that the simulation cutoff values are larger than the differences in the invariant models (except for $\triangle \mathrm{SRMR}$ ). Therefore, none of the invariant models are being flagged as "noninvariant" using the simulated cutoff values, except $\triangle$ SRMR. 
Table 13

Invariant Models' Means, Standard Deviations, and Percentiles to Yield Simulated Cutoff Values

\begin{tabular}{|c|c|c|c|c|c|c|}
\hline Index & Model & $n_{\text {Model }}$ & $M$ & $S D$ & Percentile & $n$ \\
\hline & & & & & $5^{\text {th }}$ & \\
\hline \multirow[t]{6}{*}{$\Delta$ Gamma } & Complex & 400 & -.0234 & .0027 & -.0280 & 1000 \\
\hline & & 800 & -.0236 & .0018 & -.0266 & 1000 \\
\hline & & 1200 & -.0236 & .0015 & -.0260 & 1000 \\
\hline & Simple & 400 & -.0079 & .0046 & -.0160 & 692 \\
\hline & & 800 & -.0067 & .0026 & -.0110 & 762 \\
\hline & & 1200 & -.0063 & .0018 & -.0093 & 793 \\
\hline \multirow[t]{6}{*}{$\Delta$ McDonald's } & Complex & 400 & -.1122 & .0126 & -.1334 & 1000 \\
\hline & & 800 & -.1136 & .0084 & -.1279 & 1000 \\
\hline & & 1200 & -.1136 & .0068 & -.1251 & 1000 \\
\hline & Simple & 400 & -.0389 & .0229 & -.0793 & 692 \\
\hline & & 800 & -.0332 & .0128 & -.0545 & 762 \\
\hline & & 1200 & -.0314 & .0088 & -.0459 & 793 \\
\hline \multirow[t]{7}{*}{$\Delta \mathrm{CFI}$} & Complex & 400 & -.0040 & .0006 & -.0050 & 1000 \\
\hline & & 800 & -.0041 & .0004 & -.0047 & 1000 \\
\hline & & 1200 & -.0041 & .0003 & -.0047 & 1000 \\
\hline & Simple & 400 & -.0009 & .0006 & -.0019 & 692 \\
\hline & & 800 & -.0009 & .0003 & -.0014 & 762 \\
\hline & & 1200 & -.0009 & .0002 & -.0013 & 793 \\
\hline & & & & & $95^{\text {th }}$ & \\
\hline \multirow[t]{6}{*}{$\Delta \mathrm{SRMR}$} & Complex & 400 & .8937 & .0740 & 1.0184 & 1000 \\
\hline & & 800 & .9020 & .0518 & .9883 & 1000 \\
\hline & & 1200 & .9013 & .0412 & .9710 & 1000 \\
\hline & Simple & 400 & .2279 & .0360 & .2900 & 692 \\
\hline & & 800 & .2235 & .0364 & .2660 & 762 \\
\hline & & 1200 & .2218 & .0285 & .2536 & 793 \\
\hline \multirow[t]{6}{*}{$\triangle \mathrm{RMSEA}$} & Complex & 400 & .0302 & .0058 & .0389 & 1000 \\
\hline & & 800 & .0333 & .0042 & .0388 & 1000 \\
\hline & & 1200 & .0341 & .0037 & .0389 & 1000 \\
\hline & Simple & 400 & .0155 & .0084 & .0269 & 692 \\
\hline & & 800 & .0162 & .0053 & .0234 & 762 \\
\hline & & 1200 & .0165 & .0041 & .0221 & 793 \\
\hline
\end{tabular}

Note. $n$ in the last column is the number of models without condition codes. 


\section{Power Rates}

The next analysis of the simulation study was to determine the power for each $\Delta \mathrm{GOF}$ index by condition. Power occurs when the non-invariant models are correctly identified using the simulation cutoff values from Table 12 . The $\Delta \mathrm{GOF}$ indices are calculated by subtracting the constrained non-invariant model from the unconstrained non-invariant model for each replication without condition codes. For example, the simulated cutoff value for $\Delta \mathrm{CFI}$ is -.0093 in the complex model with $n=400$. Therefore, the $\Delta$ CFI values that are subsumed under the complex model with $n=400$ and beyond the simulated cutoff value would be identified as non-invariant. The proportion of flagged non-invariant models for each condition will be the power rate of the fit indices and are in the additional supplements files. The folder labeled "COMPLEX .2" presents complex models with .2 factor loading differences, the "COMPLEX .4" folder presents complex models with .4 factor loading differences, the "SIMPLE .2" folder presents simple models with .2 factor loading differences, and the "SIMPLE .4" folder presents simple models with .4 factor loading differences.

The abbreviated model names presented in the power charts (see additional supplements) represent the different condition options for each model and are defined in Table 14. The abbreviated model names are presented so the reader will be able to identify the exact model used in each chart. For example, in the additional supplements (folder labeled "COMPLEX .2"), the power chart titled "COMPLEX_2_1200_SPECIFIC_100_40," represents the complex model (i.e., COMPLEX), .2 factor loading difference (i.e.,_2), sample size of 1200 per group (i.e., _1200), and a total of eight non-invariant factor loadings (i.e, _40: $40 \%=8$ out of 20 items were noninvariant) located on all (i.e., _100) of the specific factors only (_SPECIFIC). 
Table 14

Summary of Model Conditions

Conditions

Levels of simulation

Model Complexity ${ }^{\mathrm{a}}$

Magnitude of factor loading difference

Sample size by group

Location of non-invariant items ${ }^{b}$

Percentage of specific factors with non-invariant items $^{\text {c }}$

Percentage of total items with non-invariant items
Simple, Complex

$.2, .4$

400, 800, 12000

GEN \& SPEC, SPEC

$50 \%, 100 \%$

$20 \%, 40 \%$

Note. ${ }^{\text {a }}$ simple model: one general factor with two specific factors; complex model: one general factor with four specific factors.

${ }^{\mathrm{b}} \mathrm{GEN}$ stands for general factor and SPEC stands for specific factor

${ }^{\mathrm{c}} 50 \%$ indicates non-invariant items located on only half of the specific factors, $100 \%$ indicates non-invariant items equally located on all of the specific factors.

\section{Variance Components Analysis}

The last analysis conducted on the simulated data (presented in Table 15) was to examine the proportion of variance accounted for in each $\Delta \mathrm{GOF}$ index by simulation conditions (i.e., model complexity, magnitude of factor loading differences, sample size per group, factors with non-invariant items, percentage of specific non-invariant factors, and the percentage of total noninvariant items). The variance component analysis is useful to evaluate how much of the variability in each $\triangle \mathrm{GOF}$ index can be attributed to the different simulated conditions. The semipartial omega squared $\left(\omega^{2}\right)$ values were chosen because omega is an estimate of the population effect size and the semi-partial value is the proportion of variance explained without partialling variance out of the criterion variable (Kirk, 1995). For all of the $\triangle$ GOF indices investigated, the 
magnitude of the factor loading differences contributed the most variation to each $\Delta \mathrm{GOF}$ index except $\triangle \mathrm{SRMR}$. For $\triangle \mathrm{SRMR}$ the largest contribution of variance was model complexity (i.e., simple or complex). That is, model complexity (as evident in Tables 8 and 9 above) influences the values of $\triangle \mathrm{SRMR}$ the most (complex models had much larger $\triangle \mathrm{SRMR}$ values than the simple models). The interactions that contributed the most variance are also presented in Table 15. However, interactions with "small" variance accounted for values are suppressed. 
Table 15

Summary of Variance Component Analysis for Each $\triangle G O F$ Index

\begin{tabular}{|c|c|c|c|c|c|}
\hline Effect & $\Delta \mathrm{Gamma}$ & $\Delta \mathrm{Mc}$ & $\Delta \mathrm{CFI}$ & $\Delta \mathrm{SRMR}$ & $\triangle \mathrm{RMSEA}$ \\
\hline Model & .0527 & .0573 & .1020 & .8067 & .0377 \\
\hline Magnitude & .4448 & .4557 & .3596 & .0142 & .4126 \\
\hline$n$ & .0000 & .0000 & .0000 & .0000 & .0066 \\
\hline Location & .1005 & .1019 & .0957 & .0107 & .1011 \\
\hline Specific Percent & .0605 & .0578 & .0653 & .0020 & .0497 \\
\hline Total Percent of Items & .1477 & .1451 & .1785 & .0102 & .1327 \\
\hline Model*Specific Percent & .0114 & .0105 & .0144 & .0152 & .0088 \\
\hline Magnitude*Specific Percent & .0060 & .0036 & .0100 & .0025 & .0021 \\
\hline Magnitude*Total Percent of Items & .0274 & .0199 & .0363 & .0066 & .0136 \\
\hline Specific Percent*Total Percent of Items & .0367 & .0352 & .0391 & .0047 & .0312 \\
\hline $\begin{array}{l}\text { Model*Specific Percent*Total Percent of } \\
\text { Items }\end{array}$ & .0357 & .0392 & .0269 & .0329 & .0377 \\
\hline $\begin{array}{l}\text { Magnitude*Specific Percent*Total Percent of } \\
\text { Items }\end{array}$ & .0067 & .0048 & .0088 & .0028 & .0036 \\
\hline
\end{tabular}

Note . Model $=$ simple or complex model, Magnitude $=.2$ or .4 factor loading differences, $n=$ model sample size, Location $=$ non-invariant items on general and specific factor or just general factor, Specific Percent $=50 \%$ or $100 \%$ specific factors have non-invariant items, and Total Percent of Items $=20 \%$ or $40 \%$ of total items non-invariant. 


\section{Arkansas Benchmark Data}

Finally, the bifactor model was applied to the Arkansas Benchmark Examination data. The LR test was significant, indicating that the metric invariance test fits the data significantly worse than the configural invariance test (by group), $\Delta \chi^{2}(50)=26,421.80, p<.001$. The changes in the fit indices in Table 16 are consistent with the results of the LR test except for $\triangle$ SRMR and $\triangle$ RMSEA. The use of the empirical Arkansas Benchmark data supports the simulated cutoff values for $\Delta \mathrm{Gamma}, \Delta \mathrm{Mc}$, and $\Delta \mathrm{CFI}$.

Table 16

Summary of Fit Indices for Arkansas Benchmark Data $(n=36,391)$

\begin{tabular}{|c|c|c|c|c|c|c|}
\hline Model & $\chi^{2}(d f)$ & Gamma & Mc & CFI & SRMR & RMSEA $(90 \%$ CI) \\
\hline $\begin{array}{l}\text { Configural } \\
\text { (combined) }\end{array}$ & $\begin{array}{c}16720.334 \\
(250)\end{array}$ & .965 & .797 & .950 & .025 & $.043(.042, .043)$ \\
\hline $\begin{array}{l}\text { Configural }^{\mathrm{a}} \\
\text { (by group) }\end{array}$ & $\begin{array}{c}18583.289 \\
(500)\end{array}$ & .962 & .780 & .946 & .026 & $.045(.044, .045)$ \\
\hline \multirow[t]{2}{*}{ Metric $^{b}$} & $\begin{array}{c}45005.089 \\
(550)\end{array}$ & .911 & .543 & .867 & .158 & $.067(.066, .067)$ \\
\hline & $\Delta \chi^{2}(d f)$ & $\Delta \mathrm{Gamma}$ & $\Delta \mathrm{Mc}$ & $\Delta \mathrm{CFI}$ & $\Delta \mathrm{SRMR}$ & $\triangle \mathrm{RMSEA}$ \\
\hline $\mathrm{b}-\mathrm{a}$ & $\begin{array}{c}26,421.80 \\
(50)\end{array}$ & -.051 & -.237 & -.079 & .132 & .022 \\
\hline
\end{tabular}

Note. 18,678 boys $\& 17,713$ girls 


\section{DISCUSSION}

The purpose of this study was to evaluate the sensitivity of recommended fit indices for higher-order CFA models, more specifically, the bifactor model. The applied use of the bifactor model and, in addition, measurement invariance studies are becoming more prevalent (Gustafsson \& Balke, 1993; Chen, 2007; Cheung \& Rensvold, 2002; Luo, Petrill, \& Thompson, 1994). However, to my knowledge, there have not been any simulation studies that combine the two topics (i.e., investigating measurement invariance properties of bifactor models). Therefore, this study was designed to help fill that gap and provide applied researchers with empirical evidence as to what may or may not constitute measurement invariance in bifactor models. Several parameters were controlled including model complexity, sample size, the total number of non-invariant items, location of the non-invariant factor loading (i.e., general and specific factors), percentage of the specific factors with non-invariant items, and magnitude of factor loading differences across groups.

\section{Summary of Results}

In the results chapter, several analyses were conducted. First, the simulation cutoff values were provided for each $\Delta \mathrm{GOF}$ to calculate the Type I error and power rates. The minimum (in terms of absolute value) simulation cutoff criteria used (as shown in Table 13) to indicate a lack of invariance for $\Delta \mathrm{Gamma}$ was a value between -.026 and -.045, for $\Delta \mathrm{Mc}$ a value between -.126 to -.212 , the $\Delta$ CFI range was -.004 to -.009 , and finally for $\triangle$ SRMR and $\triangle$ RMSEA a range of .253 to .852 and .039 to .051 was used, respectively. However, based on the results of the current study, only $\Delta$ Gamma and $\Delta \mathrm{CFI}$ are recommended to use when testing for metric invariance in the bifactor model. The remaining $\triangle \mathrm{GOF}$ indices have simulated cutoff criteria that are too large, due to poor model fit when constraints were imposed, and are not recommended. In the analysis 
of Type I error rates all indices performed well (i.e., no Type I error) except $\Delta$ SRMR. In the power analysis section, $\Delta \mathrm{Gamma}$ and $\Delta \mathrm{Mc}$ had adequate power for most of the conditions, but given $\Delta \mathrm{Mc}$ large cutoff values this index is not recommended. The proportion of variance analysis revealed that the magnitude of factor loading differences contributed the most variance to $\Delta \mathrm{Gamma}, \Delta \mathrm{Mc}, \Delta \mathrm{CFI}$, and $\triangle \mathrm{RMSEA}$, whereas model complexity affected $\Delta \mathrm{SRMR}$ the most. Finally, the empirical test of the Arkansas Benchmark Examination (ABE) data provided further support of the recommended cutoff values for $\Delta \mathrm{Gamma}$ and $\Delta \mathrm{CFI}$ which were consistent with the LR tests (i.e., chi-square difference test). That is, the significant LR test indicated that the constrained model did not fit the data as well as the unconstrained model and $\Delta$ Gamma and $\Delta \mathrm{CFI}$ were beyond the cutoff criteria proposed. Furthermore, this test indicates that males and females do not perceive all of the items similarly on the Arkansas Benchmark Examination given the same ability levels.

\section{Findings/Implications}

As shown in Table 17, the cutoff values and fit indices recommended in this study vary somewhat from previous research (Chen, 2007; Cheung \& Rensvold, 2002; Meade et al., 2008) because the current study investigated an higher-order model whereas the other studies where first-order models. Chen (2007) recommends values of $\leq-.005$ to -.010 for $\Delta$ CFI, a change of $\geq$ .010 to .015 for $\triangle$ RMSEA, change of $\leq-.005$ to -.008 for $\Delta$ Gamma, a change of $\leq-.010$ to -.015

for $\Delta \mathrm{Mc}$, and a change of $\geq .025$ to .030 for $\Delta \mathrm{SRMR}$ to indicate metric invariance holds. Cheung and Rensvold (2002) also suggested invariance holds when the critical value is smaller than or equal to -.001 for $\Delta$ Gamma and $\Delta \mathrm{CFI}$ and -.02 for $\Delta \mathrm{Mc}$. Meade et al. (2008) recommended cutoff values for $\triangle \mathrm{CFI}$ as changes less than .002 and for $\Delta \mathrm{Mc}$ a value of .008 for metric invariance. However, direct comparisons of this study and their studies (Chen, 2007; Cheung \& 
Rensvold, 2002; Meade et al., 2008) are cautioned because all three studies investigated firstorder models, whereas, the current study investigated higher-order models. Nevertheless, this study, in addition to the other measurement invariance studies (e.g., Chen, 2007; Chueng \& Rensvold, 2002; Meade et al., 2008), demonstrate that fit indices can perform markedly different under certain conditions and different types of CFA models.

Table 17

Comparisons of Recommended Cutoff Criteria for $\triangle G O F$ Indices in the Bifactor Model Study Compared to Prior First-Order Studies

\begin{tabular}{lcccc}
\hline & Current Study & Chen (2007) & $\begin{array}{c}\text { Cheung \& } \\
\text { Rensvold (2002) }\end{array}$ & $\begin{array}{c}\text { Meade, Johnson, } \\
\text { \& Braddy (2008) }\end{array}$ \\
\hline$\Delta$ Gamma & -.026 to -.045 & -.005 to -.008 & -.001 & N/A \\
$\Delta$ Mc & -.126 to $-.212^{*}$ & -.025 to -.030 & -.02 & -.008 \\
$\Delta$ CFI & -.004 to -.009 & -.005 to -.010 & -.01 & -.002 \\
$\Delta$ SRMR & .253 to $.852^{*}$ & .025 to .030 & N/A & N/A \\
$\Delta$ RMSEA & .039 to $.051 *$ & .010 to .015 & N/A & N/A
\end{tabular}

Note. $* \Delta \mathrm{GOF}$ index not recommended, $\mathrm{N} / \mathrm{A}=\Delta \mathrm{GOF}$ index was not recommended by others.

Perhaps, the most influential finding of this study was the performance of $\Delta$ SRMR in both the non-invariant and invariant models. As presented in the results section, $\Delta \mathrm{SRMR}$ consistently behaved poorly in the simulation study when constraints were imposed. For example, the unconstrained model had consistent values for SRMR that could be classified as good model fit (i.e., .01 to .03). However, once the constraints were placed on the invariant models the SRMR values greatly increased. Therefore, the $\triangle \mathrm{SRMR}$ values were extremely large as well and not recommended to use when investigating the measurement invariance properties of the bifactor model. One possible explanation of SRMR's poor performance may be attributed 
to the types of constraints (i.e., general or specific factor) placed on the bifactor model. For example, if constraints are placed on the general factor, with or without constraints on the specific factor, the general factor lambdas become equal across groups, which affect every element in the main and off diagonal in the variance-covariance matrix. Therefore, the general factor lambdas act as a constant and SRMR cannot minimize the standardized residuals as well as it could without the constraints. In summary, SRMR may be too conservative to use for testing the measurement invariance properties of bifactor models and further investigation is warranted.

\section{Type I Error Summary}

In the Type I error analysis none of the $\triangle \mathrm{GOF}$ indices exhibited Type I error except $\triangle \mathrm{SRMR}$ because the non-invariant models had much larger $\Delta \mathrm{GOF}$ indices than the invariant models. Therefore, when comparing the simulated cutoff values to the baseline models' $\Delta \mathrm{GOF}$ indices, it is readily seen that none of the invariant models were flagged as "non-invariant" (i.e., no Type I error) due to the large discrepancies between the $\triangle \mathrm{GOF}$ indices of the invariant versus non-invariant models.

\section{Power Analysis Summary}

The 96 charts in Figures 5-8 presents each of the non-invariant conditions along with the power rates of each $\triangle \mathrm{GOF}$ index. For the complex models with a factor loading difference of .2, the lowest power occurred when the four non-invariant items were located only on the specific factors. As expected, conditions with smaller sample sizes had lower power rates. For the complex models with factor loading differences of .4 , all conditions and $\Delta \mathrm{GOF}$ indices had adequate power (i.e., around $80 \%$ or above) except for conditions where eight items were noninvariant and located on two out of the four (i.e., 50\%) specific factors. This occurred because 
these models would most likely not pass the test of configural invariance and therefore when testing for metric invariance the $\Delta \mathrm{GOF}$ indices do not flag the non-invariance. In these conditions the power was below $50 \%$ for all $\Delta \mathrm{GOF}$ indices. For the simple models with factor loading differences of .2 , the power was lowest when four non-invariant items were located only on the specific factors regardless of if they were on half or all of the specific factors. In addition, when sample size was 400 per group, power was also low when non-invariant items were located on both the specific and general factors with four non-invariant items. In contrast, for the simple models with factor loading differences of .4 , the power was adequate on all conditions. Only $\triangle$ RMSEA was slightly below $80 \%$ when the sample size was smallest per group (i.e., 400$)$ and four non-invariant items were located on the specific factors only.

The comparisons across models are more erratic for the small (i.e., .2) factor loading differences. When eight non-invariant items were located on all the specific factors, regardless of non-invariant loadings on only the specific factor or both the general and specific factors, both complex and simple models performed similarly. With four non-invariant items that were located equally on all the specific factors, the simple models performed worse when the non-invariant items were located on only the specific factors. When eight non-invariant items were located on half of the specific factors only, the complex models performed worse and with four noninvariant items and the same scenario (located on half of the specific factors only), both the simple and complex models performed poorly.

Comparing across models with factor loading differences of .4, the simple and complex models performed similarly (with high power rates) except when the complex models had eight non-invariant items on half of the specific factors (with non-invariant loadings on only the specific factor). In these conditions the power was lower for the complex models. This occurred 
because it is one of the worst case scenarios in the simulation (i.e., $80 \%$ of the items in a single specific factor were non-invariant) and the results are similar to Chen (2007). In Chen's 2007 study she found that once the percentage of non-invariance passes a certain threshold the changes in the GOF indices actually become smaller and therefore, the $\triangle \mathrm{GOF}$ indices do not detect the non-invariance as well. This phenomenon may occur because the models would most likely not pass the test of configural invariance due to the large discrepancies in the factor loadings between the groups. Overall, when the non-invariant loadings are located on both the specific and general factors, the $\triangle \mathrm{GOF}$ indices do a better job for detecting the non-invariance because there are more non-invariant loadings. In addition, $\Delta \mathrm{CFI}$ usually outperforms the other $\Delta \mathrm{GOF}$ indices in the simple models, but in the complex models $\Delta$ CFI does not perform as well as the other $\Delta \mathrm{GOF}$ indices (i.e., $\Delta \mathrm{Gamma}, \Delta \mathrm{Mc}$, and $\Delta \mathrm{RMSEA}$ ).

In summary, for the simple models, the $\Delta \mathrm{GOF}$ indices performed well for the .4 factor loading differences. In the simple models with .2 factor loading difference, the $\triangle \mathrm{GOF}$ indices performed poorly when four non-invariant items were located on the specific factors only. For the complex models with factor loading differences of 4 the power rates were acceptable, except for the conditions with eight non-invariant items located on only half of the specific factors. Again, this may occur because the initial model may not pass the test of configural invariance and therefore the test of metric invariance does not detect the non-invariant loadings, which is consistent with Chen's (2007) study. For the complex model with .2 factor loading differences the power rates of the $\Delta \mathrm{GOF}$ indices were more variable (i.e., inconsistent). That is, overall the $\Delta \mathrm{GOF}$ indices detect the lack of invariance less when only four items are non-invariant and located on the specific factors (regardless of if the non-invariant loadings are on half or all of the specific factors). In addition, with .2 factor loading differences in both simple and complex 
models, $\triangle$ CFI performs poorly when four non-invariant items are equally located on all the specific factors. $\triangle$ RMSEA performs poorly in all conditions when non-invariant items are located on both the specific and general factors, except when sample size is larger (i.e., 800 or above) and the eight non-invariant items are located on each of the specific factors. Overall, when the magnitude of factor loading differences were smaller (i.e., .2) all of the $\Delta$ GOF indices had lower power rates than when the factor loading differences were larger (i.e., .4).

The power rates were lower for the $\triangle \mathrm{GOF}$ indices when the non-invariant items were only located on the specific factor. In contrast, when non-invariant items were located on both the general and specific factors, the $\triangle \mathrm{GOF}$ indices can detect the lack of invariance more efficiently using maximum likelihood (ML) estimation. Mathematically, ML can provide a more "accurate" estimation when the non-invariant loadings exist on both the specific and general factors. When the factor loadings are constrained to be equal and both the general and specific factors' loadings are non-invariant, the main diagonal and associated covariances of the variance-covariance matrix has more flexibility to yield estimates of the model-implied matrix that are more closely aligned with the sample matrix. When the two matrices are more similar the residuals are minimized and the chi-square test (which evaluates exact fit between the two matrices) fits the data better which directly affects the remaining $\Delta \mathrm{GOF}$ indices (i.e., $\Delta \mathrm{Gamma}$, $\triangle \mathrm{Mc}, \Delta \mathrm{CFI}$, and $\triangle \mathrm{RMSEA}$ ) producing higher detection rates of non-invariance. Conceptually, this occurs because when both the general and specific factors' items are non-invariant the $\Delta \mathrm{GOF}$ indices have less difficulty to detect the lack of invariance because the number of noninvariant loadings is larger. Given the specific factor is the amount of covariance over and above the general factor, conditions were not generated between groups where non-invariant items existed on the general factor and invariant items existed on the specific factors. 


\section{Recommendations for Future Research}

The current study only investigated bifactor models and therefore could be extended to other types of higher-order models (i.e., higher-order models with indirect effects). For future research, further investigation of the sensitivities for each $\triangle \mathrm{GOF}$ index used in higher-order models should be evaluated, specifically SRMR. In addition, the data were simulated to be multivariate normal. Thus, future research could investigate the measurement invariance properties of higher-order models with categorical types of data and manipulating other simulation conditions as well. Recommendations for future research include using smaller sample sizes to evaluate the power of each $\Delta \mathrm{GOF}$ index. The sample sizes chosen for this study were used to ensure model convergence. However, smaller sample sizes can be simulated to try to establish a minimum sample size threshold (in general) for higher-order models. In other words, what is the minimum sample size one could possibly use before convergence issues may arise? Furthermore, since most of the formulas for the GOF indices include chi-square (i.e., chisquare directly influences other GOF indices), further work should evaluate the use of the chisquare test in first-order versus higher-order models. That is, if the chi-square test functions differently for higher- and lower-models, it will directly impact the sensitivity issues of other GOF indices as well.

\section{Conclusion}

This study demonstrates that for testing metric invariance in the bifactor models only $\Delta$ Gamma and $\Delta \mathrm{CFI}$ are recommended. Furthermore, $\triangle \mathrm{SRMR}$ performed poorly when equality constraints were placed on the factor loadings between groups and also in terms of Type I error rate and power. Future research should investigate the use of SRMR for higher-order models to evaluate if different models yield similar results along with assessing different types of 
constraints (i.e., constraining the general or specific factors). Overall, the fit indices that performed the best in terms of power were $\Delta \mathrm{Gamma}$ and $\Delta \mathrm{Mc}$, however, in most of the power conditions $\triangle \mathrm{CFI}$ and $\triangle \mathrm{RMSEA}$ performed well too. In addition, $\Delta \mathrm{Gamma}, \Delta \mathrm{Mc}, \Delta \mathrm{CFI}$, and $\triangle$ RMSEA all held Type I error to a minimum. However, the only two indices that did not have large simulated cutoff values and are recommended in this study for testing metric level invariance in the bifactor model were $\Delta$ Gamma and $\Delta$ CFI with values of -.026 to -.045 and -.004 to -.009 , respectively to indicate a lack of invariance. In the variance component analysis, the magnitude of the factor loading differences contributed the most variation to each $\Delta \mathrm{GOF}$ index except $\triangle$ SRMR, which was greatly affected by model complexity (i.e., simple versus complex models). Finally, the significant LR test in the Arkansas Benchmark Examination data was associated with values beyond the recommendations for $\Delta \mathrm{Gamma}$ and $\Delta \mathrm{CFI}$ providing further support for the suggested cutoff values. In conclusion, $\Delta \mathrm{GOF}$ indices are used for descriptive purposes only and caution must be exercised while using $\Delta \mathrm{GOF}$ indices to evaluate model fit when testing for measurement invariance properties. 


\section{REFERENCES}

Aiken, L. S., Stein, J. A., \& Bentler, P. M. (1994). Structural equation analyses of clinical subpopulation differences and comparative treatment outcomes: Characterizing the daily lives of drug addicts. Journal of Consulting and Clinical Psychology, 62, 488-499.

Anderson, J. C., \& Gerbing, D. W. (1984). The effects of sampling error on convergence, improper solutions and goodness-of-fit indices for maximum likelihood confirmatory factor analysis. Psychometrika, 49, 155-173.

Anderson, J. C., \& Gerbing, D. W. (1988). Structural equation modeling in practice: A review and recommended two-step approach. Psychological Bulletin, 103, 411-423.

Bentler, P. M. (1990). Comparative fit indices in structural models. Psychological Bulletin, 107, 238-246.

Bentler, P. M., \& Bonnet D. G. (1980). Significance tests and goodness of fit in the analysis of covariance structures. Psychological Bulletin, 88, 588-606.

Bollen, K. A. (1989). Structural equations with latent variables. New York: Wiley.

Brown, A. R., Finney, S. J., \& France, M. K. (2011). Using the bifactor model to assess the dimensionality of the hong psychological reactance scale. Educational and Psychological Measurement, 71, 170-185.

Byrne, B. M., Shavelson, R. J., \& Muthén, B. (1989). Testing for equivalence of factor covariance and mean structures: The issue of partial measurement invariance. Psychological Bulletin, 105, 456-466.

Chen, F. F. (2007). Sensitivity of goodness of fit indexes to lack of measurement invariance. Structural Equation Modeling, 14, 464-504.

Chen, F. F., West, S. G., \& Sousa, K. H. (2006). A comparison of bifactor and second-order models of quality of life. Multivariate Behavioral Research, 41, 189-225.

Cheung, G. W., \& Rensvold, R. B. (2002). Evaluating goodness-of-fit indexes for testing measurement invariance. Structural Equation Modeling, 9, 233-255.

Cohen, J. (1988) Statistical power analysis for the behavioral sciences $\left(2^{\text {nd }}\right.$ ed.). Hillsdale, NJ: Laurence Erlbaum.

Cole, D. A., Maxwell, S. E., Arvey, R., \& Salas, E. (1993). Multivariate group comparisons of variable systems: MANOVA and structural equation modeling. Psychological Bulletin, $114,174-184$.

Cudeck, R., \& Browne, M. W. (1983). Cross-validation of covariance structures. Multivariate Behavioral Research, 18, 147-167. 
DiStefano, C., \& Motl, R. W. (2006). Further investigating method effects associated with negatively worded items on self-report surveys. Structural Equation Modeling, 13, 440464.

Fan, X., \& Sivo, S. A. (2009). Using $\Delta$ Goodness-of-fit indexes in assessing mean structure invariance. Structural Equation Modeling, 16, 54-69.

Ferron, J., Dailey, R., \& Yi, Q. (2002). Effects of missspecifying the first-level error structure in two-level models of change. Multivariate Behavioral Research, 37, 379-403.

French, B., \& Finch, W. (2006). Confirmatory factor analytic procedures for the determination of measurement invariance.Structural Equation Modeling, 13, 378-402.

Gerbing, D. W., \& Anderson, J. C. (1993). Monte carlo evaluations of goodness-of-fit indices for structural equation models. In K. A. Bollen\& J. S. Long (Eds.), Testing structural equation models (40-65). Newbury Park, CA: Sage.

Green, S. B., \& Thompson, M. S. (2003). Structural equation modeling in clinical research. In M. C. Roberts \& S. S. Illardi (Eds.), Methods of research in clinical psychology: A handbook (pp. 138-175). London: Blackwell.

Grimm, K. J., \& Widaman, K. F. (2010). Residual structures in latent growth curve modeling, Structural Equation Modeling, 17, 424-442.

Gustafsson, J., \& Balke, G. (1993). General and specific abilities as predictors of school achievement. Multivariate Behavioral Research, 28, 407-434.

Hancock, G. R. (1997). Structural equation modeling methods of hypothesis testing of latent variable means. Measurement and Evaluation in Counseling and Development, 20, 91105.

Harwell, M. R. (1997). Analyzing the results of monte carlo studies in item response theory. Educational and Psychological Measurement, 57, 266-279.

Harwell, M. R., Rubinstein, E. N., Hayes, W. S., \& Olds, C. C. (1992). Summarizing Monte Carlo Results in Methodological Research: The one- and two-factor fixed effects ANOVA cases. Journal of Educational Statistics, 17, 315-339.

Hoaglin, D. C., \& Andrews, D. F. (1975). The reporting of computation-based results in statistics. The American Statistician, 29, 122-126.

Holland, P. W., \& Thayer, D. T. (1988). Differential item performance and the Mantel-Haenszel procedure. In H. Wainer and H. Braun (Eds.), Test Validity (pp .129-145). Hillsdale, NJ: Erlbaum.

Holzinger, K. J., \& Swineford, R. (1937). The bifactor method. Psychometrika, 2, 41-54.

Horn, J. L., \& McArdle, J. J. (1992). A practical and theoretical guide to measurement invariance in aging research. Experimental Aging Research, 18, 117-144. 
Hu, L., \& Bentler, P. M. (1999). Cutoff criteria for fit indexes in covariance structure analysis: Conventional criteria versus new alternatives. Structural Equation Modeling, 6, 1-55.

Jeenrich, R. I., \& Bentler, P. M. (2012). Exploratory bi-factor analysis: The oblique case. Psychometrika, 77, 442-454.

Jöreskog, K. G., \& Sörbom, D. (1981). LISREL V: Analysis of linear structural relationships by the method of maximum likelihood. Chicago: National Educational Resources.

Jöreskog, K. G., \& Sörbom, D. (1988). LISREL 7-A guide to the program and applications. ( $2^{\text {nd }}$ ed.). Chicago: SPSS.

Kirk, R. E. (1995). Experimental Design: Procedures for the behavioral sciences. ( ${ }^{\text {rd }}$ ed.).Pacific Grove, CA: Brooks/Cole.

Kline, R. B. (2011). Principles and practice of structural equation modeling.( $3^{\text {rd }}$ ed.). New York: Guilford Press.

Kwok, O., West, S. G., \& Green, S. B. (2007). The impact of misspecifying the within-subject covariance structure in multiwave longitudinal multilevel models: A monte carlo study. Multivariate Behavioral Research, 42, 557-592.

Lou, D., Petrill, S. A., \& Thompson, L. A. (1994). An exploration of genetic g: Hierarchical factor analysis of cognitive data from the western reserve twin project. Intelligence, 18 , 335-347.

Marsh, H. W., Hau, K., \& Grayson, D. (2005). Goodness of fit in structural equation models. In A. Maydeu-Olivares \&J. J. McArdle (Eds.). Contemporary Psychometrics: A Festschrift for Roderick P. McDonald (pp. 275-340). Mahwah NJ: Erlbaum.

McDonald, R. P. (1989). An index of goodness-of-fit based on noncentrality. Journal of Classification, 6, 97-103.

Meade, A. W., Johnson, E. C., \& Braddy, P. W. (2008). Power and sensitivity of alternative fitindices in tests of measurement invariance. Journal of Applied Psychology, 93, 568592.

Meredith, W. (1993). Measurement invariance, factor analysis, and factorial invariance, Psychometrika, 58, 525-543.

Meredith, W., \& Millsap, R. E. (1992). On the misuse of manifest variables in the detection of measurement bias. Psychometrika, 57, 289-311.

Millsap, R. E. (2005). Four unresolved problems in studies of factorial invariance. In A. Maydeu-Olivares \& J. J. McArdle (Eds.). Contemporary Psychometrics: A Festschrift for Roderick P. McDonald (pp. 153-171). Mahwah NJ: Erlbaum.

Millsap, R. E. (2007). Structural equation modeling made difficult. Personality and Individual Differences, 42, 875-881. 
Millsap, R. E., \& Everson, H. T. (1993). Methodology review: Statistical approaches for assessing measurement bias. Applied Psychological Measurement, 17, 297-334.

Millsap, R. E., \& Kwok, O. (2004). Evaluating the impact of partial factorial invariance on selection in two populations. Psychological Methods, 9, 93-115.

Reise, S. P., Widaman, K. F., \& Pugh, R. H. (1993).Confirmatroy factor analysis and item response theory: Two approaches for exploring measurement invariance. Psychological Bulletin, 114, 552-566.

Schmitt, N., Pulakos, E. D., \& Lieblein, A. (1984). Comparison of three techniques to assess group-level beta and gamma change. Applied Psychological Measurement, 8, 249-260.

Sivo, S., Fan, X., \& Witta, L. (2005). The biasing effects of unmodeled ARMA time series processes on latent growth curve model estimates. Structural Equation Modeling, 12,215231.

Steiger, J. H. (1989). EzPATHCausal Modeling: A supplementary module for SYSTAT and SYSGRAPH. Evanston, IL: SYSTAT.

Steiger, J. H., \& Lind, J. C. (1980, May). Statistically-based tests for the number of common factors. Paper presented at the Psychometrika Society Meeting, Iowa City.

Thompson, M. S., \& Green, S. B. (2006).Evaluating between-group differences in latent variable means. In G. R. Hancock \& R. O. Mueller (Eds.), Structural equation modeling: A second course (119-169). Greenwich, CT: Information Age.

Tucker, L. R., \& Lewis, C. (1973). The reliability coefficient for maximum likelihood factor analysis, Psychometrika, 38, 1-10.

Vandenburg, R. J. (2002). Toward a fuller understanding of and improvement in measurement invariance methods and procedures. Organizational Research Methods, 5, 139-158.

Vandenburg, R. J., \& Lance, C. E. (2000). A review and synthesis of the measurement invariance literature: Suggestions, practices, and recommendations for organizational research. Organizational Research Methods, 3, 4-69.

Yoon, M., \& Millsap, R. E. (2007). Detecting violations of factorial invariance using data-based specification searches: A monte carlo study. Structural Equation Modeling, 14, 435-463. 